NASA/TM-2010-216913

\title{
Interference-Fit Life Factors for Ball Bearings
}

Fred B. Oswald and Erwin V. Zaretsky

Glenn Research Center, Cleveland, Ohio

Joseph V. Poplawski

J.V. Poplawski \& Associates, Bethlehem, Pennsylvania 


\section{NASA STI Program . . . in Profile}

Since its founding, NASA has been dedicated to the advancement of aeronautics and space science. The NASA Scientific and Technical Information (STI) program plays a key part in helping NASA maintain this important role.

The NASA STI Program operates under the auspices of the Agency Chief Information Officer. It collects, organizes, provides for archiving, and disseminates NASA's STI. The NASA STI program provides access to the NASA Aeronautics and Space Database and its public interface, the NASA Technical Reports Server, thus providing one of the largest collections of aeronautical and space science STI in the world. Results are published in both non-NASA channels and by NASA in the NASA STI Report Series, which includes the following report types:

- TECHNICAL PUBLICATION. Reports of completed research or a major significant phase of research that present the results of NASA programs and include extensive data or theoretical analysis. Includes compilations of significant scientific and technical data and information deemed to be of continuing reference value. NASA counterpart of peer-reviewed formal professional papers but has less stringent limitations on manuscript length and extent of graphic presentations.

- TECHNICAL MEMORANDUM. Scientific and technical findings that are preliminary or of specialized interest, e.g., quick release reports, working papers, and bibliographies that contain minimal annotation. Does not contain extensive analysis.

- CONTRACTOR REPORT. Scientific and technical findings by NASA-sponsored contractors and grantees.
- CONFERENCE PUBLICATION. Collected papers from scientific and technical conferences, symposia, seminars, or other meetings sponsored or cosponsored by NASA.

- SPECIAL PUBLICATION. Scientific, technical, or historical information from NASA programs, projects, and missions, often concerned with subjects having substantial public interest.

- TECHNICAL TRANSLATION. Englishlanguage translations of foreign scientific and technical material pertinent to NASA's mission.

Specialized services also include creating custom thesauri, building customized databases, organizing and publishing research results.

For more information about the NASA STI program, see the following:

- Access the NASA STI program home page at http://www.sti.nasa.gov

- E-mail your question via the Internet to help@ sti.nasa.gov

- Fax your question to the NASA STI Help Desk at $443-757-5803$

- Telephone the NASA STI Help Desk at 443-757-5802

- Write to: NASA Center for AeroSpace Information (CASI) 7115 Standard Drive Hanover, MD 21076-1320 
NASA/TM-2010-216913

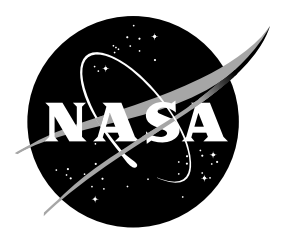

\section{Interference-Fit Life Factors for Ball Bearings}

Fred B. Oswald and Erwin V. Zaretsky

Glenn Research Center, Cleveland, Ohio

Joseph V. Poplawski

J.V. Poplawski \& Associates, Bethlehem, Pennsylvania

National Aeronautics and

Space Administration

Glenn Research Center

Cleveland, Ohio 44135 
Trade names and trademarks are used in this report for identification only. Their usage does not constitute an official endorsement, either expressed or implied, by the National Aeronautics and Space Administration.

Level of Review: This material has been technically reviewed by technical management.

Available from

NASA Center for Aerospace Information 7115 Standard Drive

Hanover, MD 21076-1320
National Technical Information Service 5301 Shawnee Road Alexandria, VA 22312

Available electronically at http://gltrs.grc.nasa.gov 


\title{
Interference-Fit Life Factors for Ball Bearings
}

\author{
Fred B. Oswald and Erwin V. Zaretsky \\ National Aeronautics and Space Administration \\ Glenn Research Center \\ Cleveland, Ohio 44135 \\ Joseph V. Poplawski \\ J.V. Poplawski \& Associates \\ Bethlehem, Pennsylvania 18018
}

\begin{abstract}
The effect of hoop stresses on the rolling-element fatigue life of angular-contact and deep-groove ball bearings was determined for common inner-ring interference fits at the ABEC-5 tolerance level. The analysis was applied to over 1150 bearing configurations and load cases. Hoop stresses were superimposed on the Hertzian principal stresses created by the applied bearing load to calculate the inner-race maximum shearing stress. The resulting fatigue life of the bearing was recalculated through a series of equations. The reduction in the fatigue life is presented as life factors that are applied to the unfactored bearing life. The life factors found in this study ranged from 1.00 (no life reduction) - where there was no net interface pressure - to a worst case of 0.38 (a 62-percent life reduction). For a given interference fit, the reduction in life is different for angular-contact and deep-groove ball bearings. Interference fits also affect the maximum Hertz stress-life relation. Experimental data of Czyzewski, showing the effect of interference fit on rolling-element fatigue life, were reanalyzed to determine the shear stress-life exponent. The Czyzewski data shear stress-life exponent $c$ equals 8.77, compared with the assumed value of 9 . Results are presented as tables and charts of life factors for angular-contact and deep-groove ball bearings with light, normal, and heavy loads and interference fits ranging from extremely light to extremely heavy.
\end{abstract}

\section{Introduction}

Rolling-element bearings often utilize a tight interference fit between the bore of the bearing inner ring and shaft and/or between the outer diameter of the bearing ring and housing bore to prevent fretting damage at the respective interfaces. American National Standards Institute/American Bearing Manufacturers Association (ANSI/ABMA) standards (ANSI/ABMA-7 and -20 (Refs. 1 to 2)) as well as catalogs of bearing manufacturers specify suggested fits for various operating conditions. Any fit must be based on the most severe operating conditions expected, including the highest speeds and highest vibration levels.

A tight fit of the bearing inner ring over the shaft reduces internal bearing clearance. Initial internal clearance can be added to the bearing to compensate for the clearance change. In addition, the interference fit of the inner ring over the shaft adds a hoop stress on the bearing inner ring. Czyzewski (Ref. 3) first showed that tensile hoop stresses induced in the bearing inner ring can negatively affect rolling-element fatigue life.
Coe and Zaretsky (Ref. 4) analyzed the effect of these hoop stresses on rolling-element fatigue life. Their work was based on the analysis of Hertzian principal stresses from Jones (Ref. 5) and the Lundberg-Palmgren bearing life theory (Lundberg and Palmgren (Ref. 6)). Coe and Zaretsky (Ref. 4) superimposed the hoop stresses on the Hertzian principal stresses, whereby the shearing stresses in the stressed volume below the contact between the rolling element (ball or roller) and inner race of the bearing increase. The increased maximum shearing stress at a depth $z$ below the contacting surface due to hoop stress is

$$
\left(\tau_{\max }\right)_{h}=\tau_{\max }-\frac{\sigma_{h}}{2}
$$

where $\tau_{\max }$ (a negative quantity) is the maximum shearing stress, $\sigma_{h}$ (a positive quantity for the inner ring) is the hoop stress at the depth of the maximum shearing stress, and $\left(\tau_{\max }\right)_{h}$ is the maximum shearing stress including the effect of the hoop stress.

Where bearing inner rings are manufactured from casecarburized steel, there are compressive residual stresses induced in the raceway. There may also be compressive residual stresses induced by the heat-treating process in through-hardened steels. The presence of these residual stresses may offset the tensile stresses created by the interference fit. Equation (1a) can be modified to account for residual stress as follows:

$$
\left(\tau_{\max }\right)_{h}=\tau_{\max }-\frac{1}{2}\left(\sigma_{h}+\sigma_{r}\right)
$$

where $\sigma_{r}$ is a negative quantity for compressive stress. As a result, the detrimental effect of an interference fit on bearing life can be mitigated by the presence of compressive residual stresses in the bearing ring (Zaretsky (Ref. 7)).

The life of the race is inversely proportional to the maximum shearing stress including (tensile) hoop stress and residual stress to an exponent $c$ :

$$
L \sim \frac{1}{\left|\tau_{\max }\right|_{h}^{c}}
$$

Zaretsky (Ref. 7) and Zaretsky et al. (Ref. 8) developed a procedure (Zaretsky's Rule) for separating the lives of the 
rolling element set from the lives the of bearing races. This provides an improvement on the prior Coe and Zaretsky analysis (Ref. 4), that had, for simplicity, assumed that all bearing components are equally affected by the inner-race interference fit. By using Zaretsky's Rule, the analysis applies the reduction in life only to the inner race without modifying the computed lives of the outer race or the rolling elements.

Zaretsky et al. (Ref. 9) performed a finite element analysis on the inner ring of an angular-contact ball bearing to determine the radius of an equivalent cylindrical race. They then analyzed 45- and 120-mm angular-contact ball bearings as though the inner rings were cylindrical to calculate the hoop stress. By applying Zaretsky's Rule, they calculated the life adjusted for heavy interference fits and inertial effects for bearings used in high-speed jet engines.

Oswald et al. (Ref. 10) applied this method to cylindrical roller bearings to calculate life factors for interference fit in roller bearings and to show the effect of interference fit on the relationship between life and the maximum Hertz stress. Heavy interference fits on the inner ring of a cylindrical roller bearing were found to significantly reduce bearing fatigue life. For example, the tightest fit at the high end of the tolerance band produced a life reduction of approximately 60 percent for an inner-race maximum Hertz stress of $1200 \mathrm{MPa}(175 \mathrm{ksi})$ in comparison to the life of a bearing without interference fit.
In view of the aforementioned, the objectives of this work were to expand the analysis of Oswald et al. (Ref. 10) and Zaretsky et al. (Ref. 9) to (1) calculate the reduction in fatigue life due to the interference fit of the inner ring for several classes of radially loaded deep-groove ball and thrust-loaded angular-contact ball bearings; (2) independently determine the lives of the inner races, outer races, and ball sets subject to inner-ring interference fit; and (3) develop life factors for interference fits that can be applied to the bearing life calculation according to the ANSI/ABMA standards for shaft fitting (Refs. 1 to 2).

\section{Enabling Equations}

\section{Subsurface Shearing Stresses}

A representative deep-groove ball bearing is shown in Figure 1 (a) and (b). The bearing comprises an inner and outer ring and a plurality of balls interspersed between the two rings and positioned by a cage or separator. The angular-contact ball bearing shown in cross section in Figure 1(c) is similar to the deep-groove bearing except that the race shoulders are not symmetrical; a shoulder on one or both of the rings is generally relieved (removed) on one side.

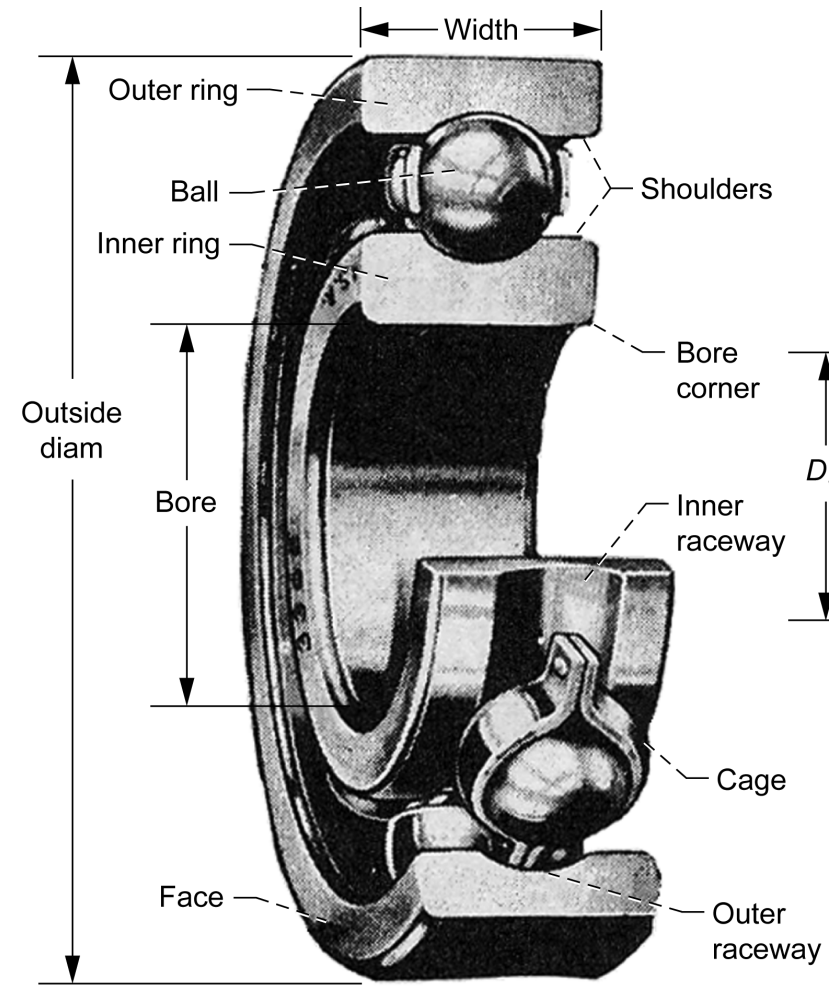

(a)

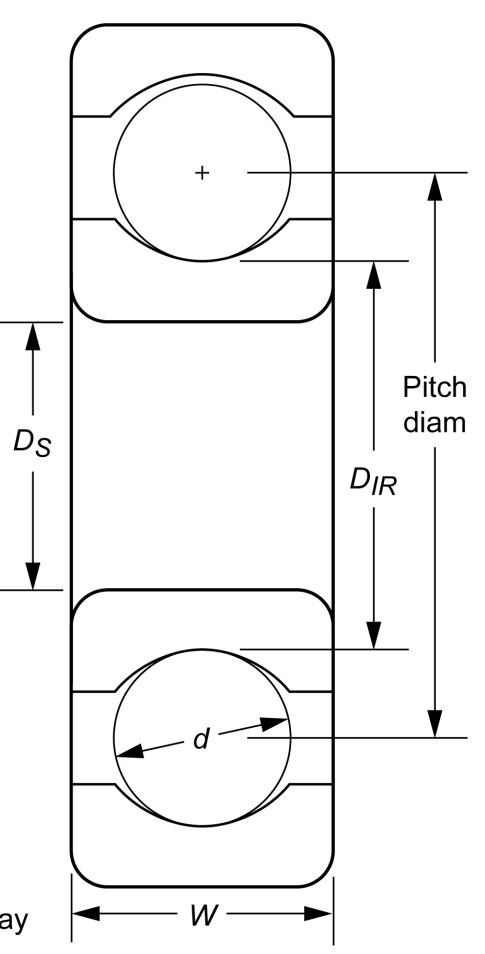

(b)

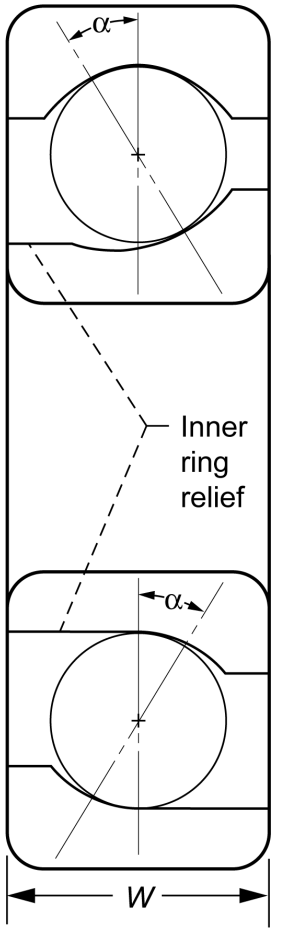

(c)

Figure 1.-Ball bearing details. (a) Deep-groove ball bearing. (b) Deep-groove bearing cross section. (c) Angularcontact bearing cross section. $D_{S}$, diameter of shaft; $D_{I R}$, diameter of inner race of bearing; $d$, diameter of ball; $W$, width of bearing ring; $\alpha$, contact angle. 


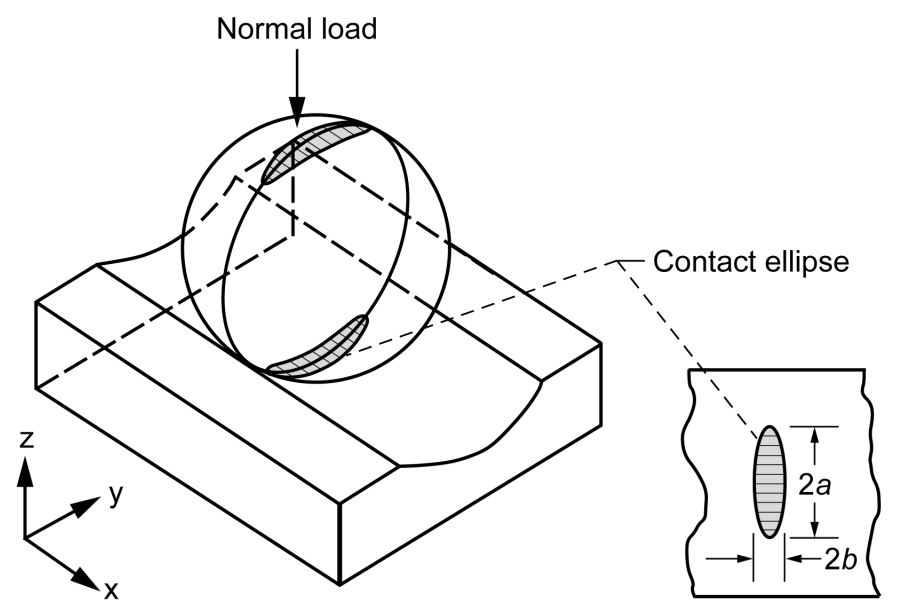

Figure 2.-Schematic of contact profile of ball on raceway; $a$ and $b$, semiwidths of major and minor axes of Hertzian contact area, respectively.

Figure 2 is a schematic of the contact profile of a ball on a race. Figure 3(a) shows the surface (Hertz) stress distribution under the ball and the principle stresses at $z$, a critical location below the surface. Figure 3(b) shows the stress distribution below the surface. From these principal stresses, the shearing stresses can be calculated.

Three shearing stresses can be applied to bearing life analysis: the orthogonal, the octahedral, and the maximum. For the analysis reported herein, only the maximum shearing stress is considered. The maximum shearing stress is one-half the maximum difference between the principal stresses:

$$
\tau_{\max }=\frac{\sigma_{z}-\sigma_{x}}{2}
$$

Note that with the sign convention used here $\tau_{\max }$ is negative.

From Jones (Ref. 5), the maximum subsurface shearing stress $\tau_{\max }$ below a rolling element is proportional to the maximum Hertz stress $S_{\max }$ :

$$
\tau_{\max }=-k_{1} S_{\max }
$$

where $k_{1}$ is a conversion constant and $S_{\max }$ is positive.

Oswald et al. (Ref. 10), based on earlier work by Coe and Zaretsky (Ref. 4), showed that in a cylindrical roller bearing the maximum subsurface shear stress due to Hertzian loading is $-0.300 S_{\max }$ and that this stress occurs at a nondimensional depth $u$ below the surface, where $u=z / b=0.786$ and $b$ is the semiwidth in the direction of rolling of the rectangular contact zone beneath the most heavily loaded roller. This result is consistent with Figure 4 (from Jones (Ref. 5)) for an inverseellipticity ratio $b / a=0$.

The principal stresses in the normal and tangential direction for a ball bearing and the effect of the added hoop stress are illustrated in Figure 5, where $S_{n}$ is the normal stress, $S_{t}$ is the tangential stress in the direction of rolling due only to Hertzian

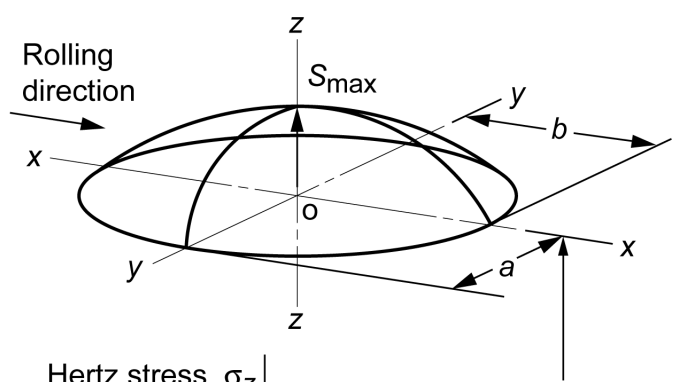

Hertz stress, $\sigma_{z}$

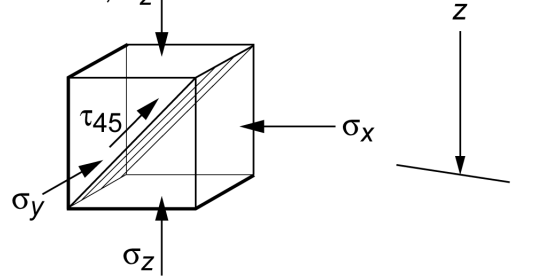

Values of stress components along centerline (see Fig. 3(a)) normalized to $\sigma_{z}$ maximum

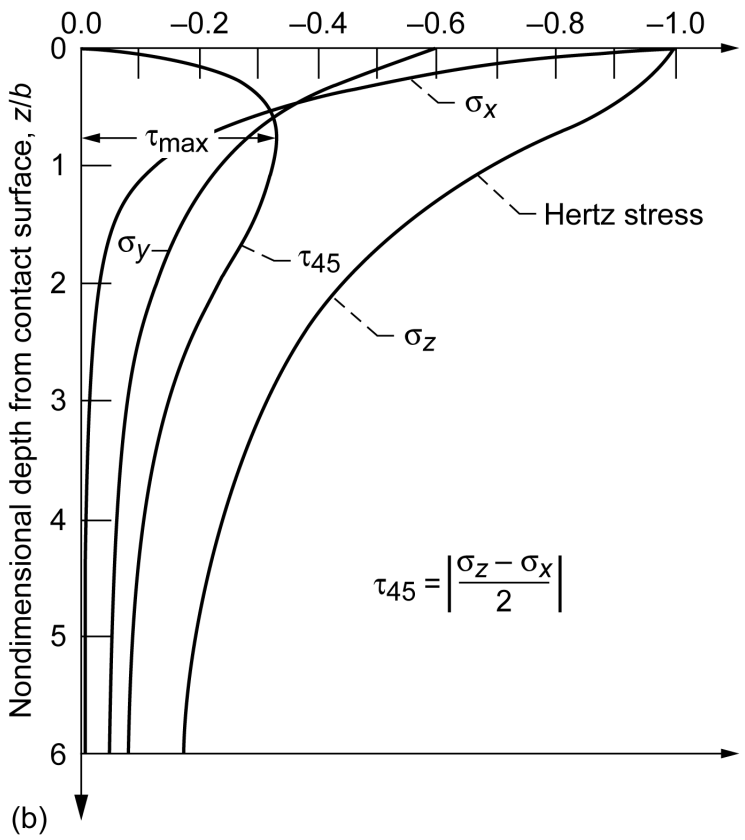

Figure 3.- Subsurface stress field under point contact. (a) Hertz stress distribution for ball on raceway showing principal stresses ( $\sigma$, stress; $\tau$, shear stress; and $S_{\max }$, maximum Hertz stress) at depth $z$ below surface. (b) Distribution of principal and shearing stress as function of depth $z / b$ below surface.

loading, and $S_{t}^{\prime}$ is the hoop stress superimposed on $S_{t}$. The maximum shear stress is one-half the difference between $S_{n}$ and $S_{t}$.

In a ball bearing, the contact area is elliptical. The solution for the location and magnitude of the maximum subsurface shear stress involves calculating the size of both axes of the contact ellipse, which requires evaluation of elliptic integrals. 
Jones (Ref. 5) provides a procedure for estimating the size of the contact ellipse and the location and magnitude of the maximum subsurface shear stress by looking up parameters in charts such as Figure 4.

Brewe and Hamrock (Ref. 11) and Hamrock and Brewe (Ref. 12) provide curve fits for estimating the contact ellipse. Antoine et al. (Ref. 13) provide a more accurate, but slightly more complicated, procedure. Bearing analysis software typically evaluates these parameters through iteration.

Zaretsky (Ref. 7) and Oswald et al. (Ref. 10) give a simplified procedure for finding the effect due to hoop stress from inner-ring interference fits on a cylindrical roller bearing. This simplified procedure, when used to calculate the resulting life of a roller bearing, gives results within 1 percent of the value found by iterating for the actual location of the maximum shear stress, even with a very heavy fit. With a few modifications, this procedure can also be applied to ball bearings.

Zaretsky's procedure (Ref. 7) requires the contact pressure $p_{i}$ between the inner ring and the shaft. For the case of a bearing ring shrunk on a solid shaft where both components have the same material properties, $p_{i}$ can be found from Equation (5) (adapted from Juvinall (Ref. 14)), where $E$ is Young's modulus, $\Delta$ is the diametral interference, $D_{S}$ is the common diameter of the shaft and bearing bore, and $D_{\text {eff }}$ is the effective outside diameter of the inner ring.

$$
p_{i}=\frac{E \Delta\left(D_{\mathrm{eff}}^{2}-D_{S}^{2}\right)}{2 D_{S} D_{\mathrm{eff}}^{2}}
$$

The effective inner-ring outside diameter for a ball bearing is calculated by adding the area of the shoulders of the bearing race to the "shoulderless" inner race and dividing by the width of the ring as shown in Figure 6(a) and (b). For the typical shoulder height of 20 percent of the ball diameter $d$, the sector angle $\theta=1.854$ radians $\left(106.24^{\circ}\right)$, as shown in Figure 6(c).

$$
\theta / 2=\cos ^{-1}\left(\frac{0.3 d}{0.5 d}\right) \Rightarrow \theta=1.854
$$

The area removed in grinding the ball track is

$$
A_{\text {sector }}=\frac{R^{2}}{2}(\theta-\sin \theta)=0.1118 d^{2}
$$

where $R$ is the radius of curvature, and the effective diameter for a deep-groove ball bearing is

$$
D_{\text {eff }}=D_{I R}+0.2 d-\frac{A_{\text {sector }}}{W}
$$

where $D_{I R}$ is the diameter of the inner race and $W$ is the width of the ball bearing ring.

On an angular-contact ball bearing, the shoulder is generally relieved (removed) on one side (see (Fig. 6(b)); hence the

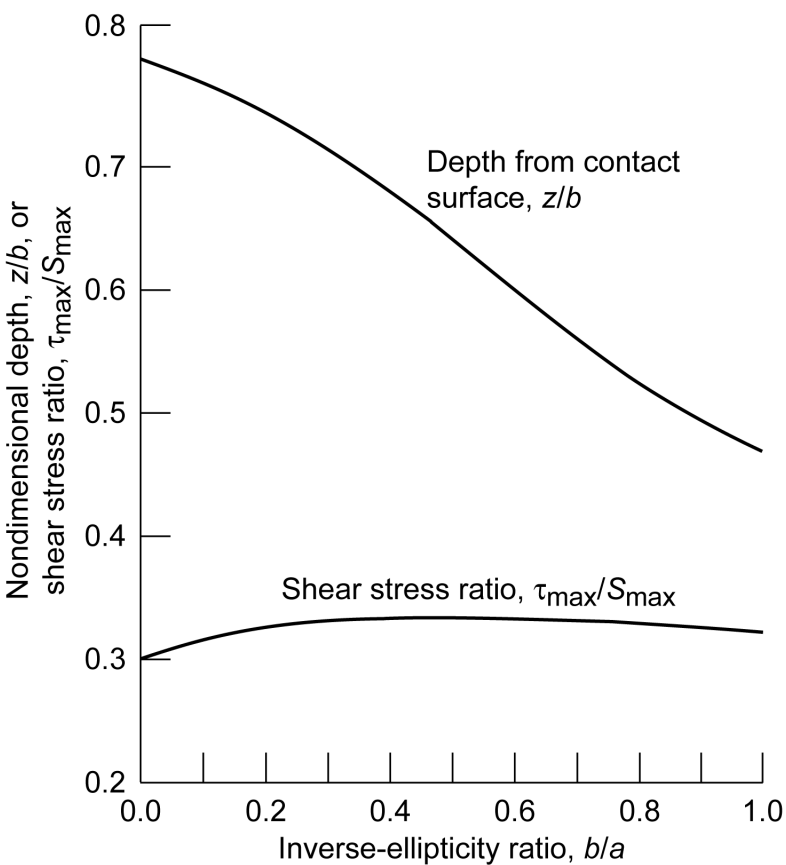

Figure 4.-Plot of nondimensional depth, $z / b$ from contact surface and shear stress ratio, $\tau_{\max } / S_{\max }$ versus inverse-ellipticity ratio, b/a (from, Jones, (Ref. 5)).

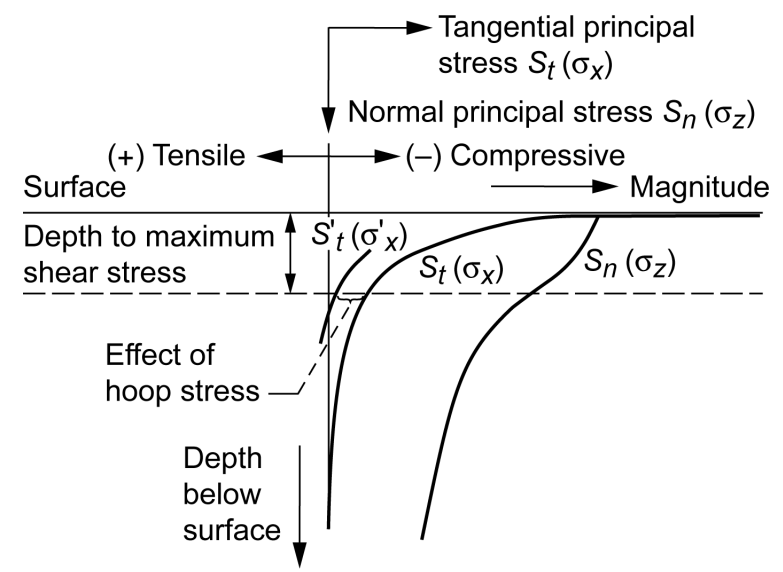

Figure 5.-Effect of superimposing hoop stress on tangential principal stress in direction of rolling under Hertzian contact. $S_{t}^{\prime}$, tangential stress including hoop stress.

second and third terms on the right-hand side of Equation (8a) are divided by 2 .

$$
D_{\text {eff }}=D_{I R}+0.1 d-\frac{A_{\text {sector }}}{2 W}
$$

However, if the inner race shoulder is not relieved, Equation (8a) should be used. 


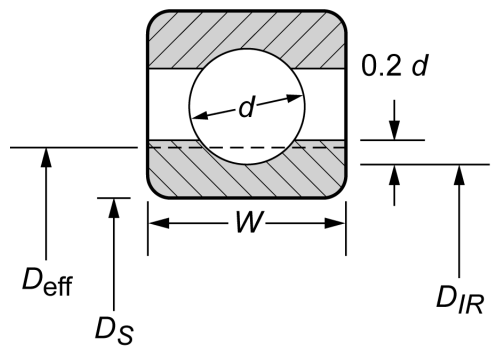

(a)

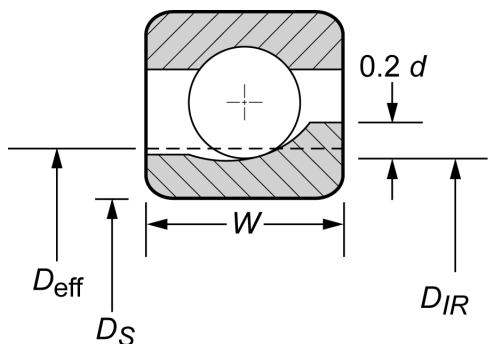

(b)

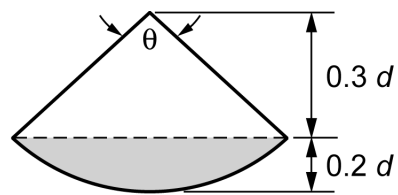

(c)

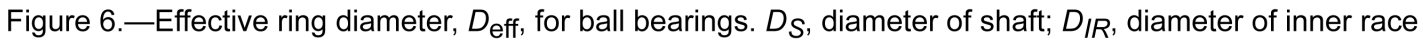
of bearing; $W$, width of bearing ring, $d$, diameter of ball. (a) Deep-groove ball bearing. (b) Angular-contact ball bearing. (c) Area of sector representing material removed from ball track.

\section{Determining Hoop Stress at the Location of Maximum Subsurface Shearing Stress}

Equation (9), adapted from Juvinall (Ref. 14), gives the tangential (hoop) stress in a bearing ring shrunk on a solid shaft. This equation is based on the stress in an internally pressurized, thick-wall cylinder where the shaft and ring are made from the same material-

$$
\sigma_{h}=\frac{p_{i} D_{S}^{2}}{D_{\text {eff }}^{2}-D_{S}^{2}}\left[1+\left(\frac{D_{\text {eff }}}{D}\right)^{2}\right]
$$

where $\sigma_{h}$ is the tangential (hoop) stress, $p_{i}$ is the internal pressure from Equation (5), $D_{S}$ is the common diameter of the shaft and bearing bore, $D_{\text {eff }}$ is the effective thick-wall cylinder diameter of the inner ring from Equations (8a) or (8b), and $D$ is the diameter at the location of the maximum shear stress beneath the surface of the inner race.

\section{Strict Series Reliability and Zaretsky's Rule}

Lundberg and Palmgren (Ref. 6) first derived the relationship between individual rolling-element bearing component lives and system life. A bearing is a system of multiple components, each with a different life. As a result, the life of the system is different from the life of an individual component in the system. The fatigue lives of each of the bearing components are combined to calculate the system $L_{10}$ life using strictseries system reliability (Lundberg and Palmgren (Ref. 6)) and the two-parameter Weibull distribution function (Weibull (Refs. 15 to 17)) for the bearing components comprising the system. The resultant life of the bearing cannot be longer than the life of the bearing component with the shortest life. Lundberg and Palmgren (Ref. 6) express the bearing system fatigue life at a given reliability, $S$, as follows:

$$
\frac{1}{L^{e}}=\frac{1}{L_{I R}^{e}}+\frac{1}{L_{O R}^{e}}
$$

where $L$ is life and $L_{I R}$ and $L_{O R}$ are the lives of the inner and outer races, respectively.

Zaretsky et al. (Ref. 8) note that the life of the rollingelement set is implicitly included in the inner- and outer-race lives in Equation (10). If the life of the balls $L_{\mathrm{B}}$ (taken as a set) is separated from the race lives, Equation (10) can be rewritten as

$$
\frac{1}{L^{e}}=\frac{1}{L_{I R-a d j}^{e}}+\frac{1}{L_{B}^{e}}+\frac{1}{L_{O R-a d j}^{e}}
$$

where $a d j$ in the subscript indicates adjusted race lives that are greater than the corresponding lives in Equation (10). Zaretsky (Ref. 7) and Zaretsky et al. (Ref. 8) observe that $L_{O R}$ is generally greater than $L_{I R}$.

For a deep-groove bearing, $L_{B}$ is equal to or greater than $L_{O R}$. Herein, it is assumed that, for a deep-groove bearing, $L_{B}=L_{O R}$; thus, Equation (11) becomes

$$
\frac{1}{L^{e}}=\frac{1}{L_{I R-a d j}^{e}}+\frac{2}{L_{O R-a d j}^{e}}
$$

For an angular-contact ball bearing, $L_{B}$ is equal to or greater than $L_{I R}$. Herein, it is assumed that, for an angular-contact bearing, $L_{B}=L_{I R}$; thus, Equation (11) becomes

$$
\frac{1}{L^{e}}=\frac{2}{L_{I R-a d j}^{e}}+\frac{1}{L_{O R-a d j}^{e}}
$$

The ratio of the lives of the outer and inner races is defined as

$$
X=\frac{L_{O R}}{L_{I R}}
$$

If the life ratio $X$ does not change when the ball set life is separated from the race lives, Equation (12a) for deep-groove bearings becomes 


$$
\frac{1}{L^{e}}=\frac{1}{L_{I R-a d j}^{e}}+\frac{2}{\left(X \cdot L_{I R-a d j}\right)^{e}}
$$

Likewise, for angular-contact bearings, Equation becomes

$$
\frac{1}{L^{e}}=\frac{2}{L_{I R-a d j}^{e}}+\frac{1}{\left(X \cdot L_{I R-a d j}\right)^{e}}
$$

\section{Bearing Life Factor for Interference Fit}

Coe and Zaretsky (Ref. 4) assumed that $L R$, the life ratio for the hoop stress on the inner race, is the 9th power of the ratio of $\tau_{\max }$ (from Eq. (4)) to $\left(\tau_{\max }\right)_{h}$.

$$
L R=\frac{(L)_{h}}{L}=\left[\frac{\tau_{\max }}{\left(\tau_{\max }\right)_{h}}\right]^{9}
$$

For roller bearings, $\left(\tau_{\max }\right)_{h}$ can be computed from the simplified procedure of Zaretsky (Ref. 7); and for ball bearings, $\left(\tau_{\max }\right)_{h}$ can be computed from a modified version of the simplified procedure.

Equation (15) is based on earlier work by Lundberg and Palmgren (Ref. 6) that uses life exponents for shear-stress that range from 6.9 to 9.3. An exponent of 9 is assumed for the current work. For further discussion of life exponents, see Poplawski et al. (Ref. 18) and the discussion later in this article.

Coe and Zaretsky (Ref. 4) applied the life ratio to the life of the entire bearing, which produces an overly conservative estimate for the life of the bearing. Herein, the life ratio is applied only to the inner race. This new value for the inner-race life $L R \cdot L_{I R-a d j}$ is used in the first term on the right-hand side of Equation (14a) to calculate the life of a deep-groove bearing $(L)_{h}$, including the effects of Hertzian loading and hoop stress. Equation (16a) is identical to the equation developed for the life of a cylindrical roller bearing in Oswald et al. (Ref. 10).

$$
\frac{1}{(L)_{h}^{e}}=\frac{1}{\left(L R \cdot L_{I R-a d j}\right)^{e}}+\frac{2}{\left(X \cdot L_{I R-a d j}\right)^{e}}
$$

Likewise, for angular-contact bearings, Equation (14b) is modified to include the effects of Hertzian loading and hoop stress:

$$
\begin{aligned}
\frac{1}{(L)_{h}^{e}}=\frac{1}{\left(L R \cdot L_{I R-a d j}\right)^{e}} & \begin{array}{r}
\quad \frac{1}{L_{I R-a d j}^{e}}+\frac{1}{\left(X \cdot L_{I R-a d j}\right)^{e}}
\end{array}
\end{aligned}
$$

Finally, the life factor for hoop stress $(L F)_{h}$ is computed as the ratio of $(L)_{h}$ divided by the original life of the bearing $L$ :

$$
(L F)_{h}=\frac{(L)_{h}}{L}
$$

\section{Determining Maximum Hertz Stress on the Basis of Static Radial Load Capacity}

Rolling-element bearing static load capacity was first defined in terms of deformation by Palmgren (Ref. 19): “... the allowable permanent deformation of rolling element and bearing ring [race] at a contact as 0.0001 times the diameter of the rolling element...." For ball bearings, this corresponds to a maximum Hertz stress of $4000 \mathrm{MPa}$ (580 ksi). From Hertz theory, Jones (Ref. 5) shows that the relationship between maximum Hertz stress and radial load $P$ for a cylindrical roller bearing is

$$
S_{\max } \sim \sqrt{P}
$$

For a ball bearing, the corresponding relationship is

$$
S_{\max } \sim \sqrt[3]{P}
$$

Nearly all bearing manufacturers' catalogs provide the static radial load capacity $C_{o r}$ for any bearing size. Hence, to determine the appropriate stress at the applied radial load on a ball bearing inner race, Equation (19) can be rewritten as follows:

$$
S_{\max }=\mathrm{k}_{2}\left[\frac{P}{C_{o r}}\right]^{1 / 3}
$$

where the conversion constant $k_{2}=4000$ for SI units with $S_{\max }$ expressed in megapascals, or $k_{2}=580$ for English traditional units with $S_{\max }$ expressed in kips per square inch (ksi). Table 1, which uses bearing catalog data from The Timken Company (Ref. 20), gives the static radial load capacity $C_{o r}$ for the bearings discussed herein. Figure 7 shows $S_{\max }$ plotted as a function of static load capacity from Equation (20). The appropriate life factor can be interpolated from Tables 2 to 5 for the various interference fits.

The value of $k_{2}=4000 \mathrm{MPa}$ (580 ksi) is implicitly included in the static load ratings in the 1978 revision of ANSI/ABMA standard 9 (ANSI/AFBMA-Std 9 (Ref. 21)). In the most recent (2000) revision (ANSI/ABMA-9 (Ref. 22)), this permissible stress has been increased by 5 percent to $4200 \mathrm{MPa}$ (609 ksi).The corresponding ISO standard, (ISO 76 (Ref. 23)) also specifies the larger value, $4200 \mathrm{MPa}$. Herein $k_{2}=4000$ was used because it agrees better with the catalog values that were used. 


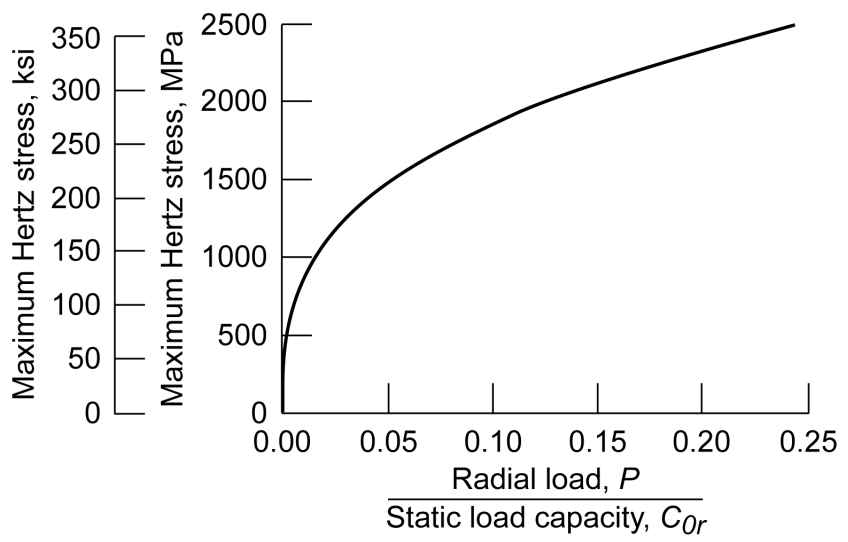

Figure 7.-Relationship between ball bearing maximum Hertz stress and $P / C_{O r}$

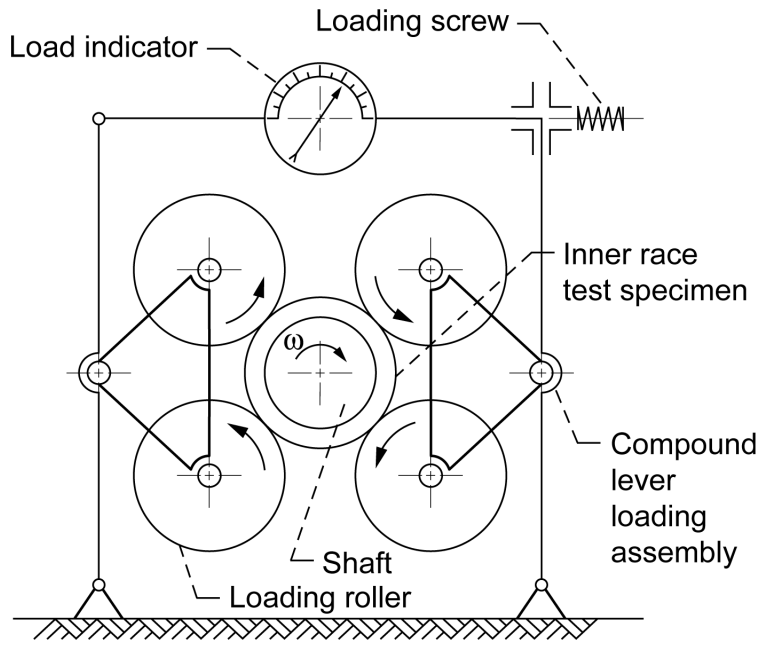

Figure 8.-Czyzewski's rolling-contact test machine; adapted from Czyzewski (Ref. 25).
As an example of using this procedure, consider a 210 -size deep-groove ball bearing with a radial load $P=1847 \mathrm{~N}$ (415.1 lbf). From Table 1, $C_{\mathrm{or}}=23162 \mathrm{~N}(5207 \mathrm{lbf})$. Using either Figure 7, with $P / C_{\mathrm{or}}=0.0797$, or Equation (20), with $\sqrt[3]{P / C}$ or $=0.43043$, and the appropriate value for $k_{2}$ yields $S_{\max }=1722 \mathrm{MPa}(249.8 \mathrm{ksi})$. The bearing analysis code (Poplawski et al. (Ref. 24)) calculated $S_{\max }=1720 \mathrm{MPa}$ (249.5 ksi).

For most low-speed ball bearing applications (less than 1 million $D N$, where $D N$ is the inner-ring speed in rpm multiplied by the bearing bore diameter in millimeters), the determination of the appropriate life factor based on bearing size, radial load, and interference fit can be related to $C_{\mathrm{o}}$ without the need to perform extensive calculations.

\section{Czyzewski's Experiment}

Czyzewski (Ref. 3) performed rolling-element fatigue tests on modified inner-races from NU 209 roller bearings. These tests show the effect of interference fits between the inner-race and the shaft of his test machine. For all the Czyzewski tests, the stressed volume calculated according to Lundberg and Palmgren (Ref. 6) and the maximum Hertz (contact) stress remained unchanged.

The test apparatus, shown in Figure 8 (redrawn from Czyzewski (Ref. 25)), employs four loading rollers through a compound lever mechanism. The inner-race test article has a $55-\mathrm{mm}$ outside diameter, $45-\mathrm{mm}$ bore, and $19-\mathrm{mm}$ length. The size of the loading rollers is not given; however from Figure 8, they apparently have the same diameter as the test races, $55 \mathrm{~mm}$.

The test races were made from an unspecified bearing steel annealed to a Brinell hardness of 170 HB (Czyzewski (Ref. 25)) (approximately Rockwell B 86 or less than Rockwell C 15). (We assume that the races were made from 52100 steel tempered from Rockwell C 60.) The races had a transverse crown of 50-mm radius with 5-mm flat length.

TABLE 1.-DEEP-GROOVE AND ANGULAR-CONTACT BALL BEARING PROPERTIES

\begin{tabular}{|c|c|c|c|c|c|c|c|c|c|c|c|}
\hline \multirow[t]{2}{*}{$\begin{array}{c}\text { Bearing } \\
\text { size }\end{array}$} & \multirow[t]{2}{*}{$\begin{array}{c}\text { Bore, } \\
\mathrm{mm}\end{array}$} & \multirow[t]{2}{*}{$\begin{array}{c}\text { Outside } \\
\text { diameter, } \\
\text { mm }\end{array}$} & \multirow[t]{2}{*}{$\begin{array}{c}\text { Number } \\
\text { of } \\
\text { balls }\end{array}$} & \multicolumn{2}{|c|}{$\begin{array}{c}\text { Ball diameter, } \\
d\end{array}$} & \multicolumn{2}{|c|}{$\begin{array}{c}\text { Inner-race outside } \\
\text { diameter, } \\
D_{I R}\end{array}$} & \multicolumn{2}{|c|}{$\begin{array}{c}\text { Static radial load } \\
\text { capacity, } \\
C_{\mathrm{o}} r\end{array}$} & \multicolumn{2}{|c|}{$\begin{array}{c}\text { Dynamic radial } \\
\text { load capacity, } \\
C_{r}\end{array}$} \\
\hline & & & & $\mathrm{mm}$ & in. & $\mathrm{mm}$ & in. & $\mathrm{N}$ & lbf & $\mathrm{N}$ & $\mathrm{lbf}$ \\
\hline 1906 & 30 & 47 & 14 & 4.7625 & $3 / 16$ & 33.738 & 1.3282 & 5009 & 1126 & 7242 & 1628 \\
\hline 206 & & 62 & 9 & 9.525 & $3 / 8$ & 36.475 & 1.4360 & 11254 & 2530 & 19457 & 4374 \\
\hline 1910 & 50 & 72 & 17 & 6.35 & $1 / 4$ & 54.65 & 2.1516 & 11152 & 2507 & 13398 & 3012 \\
\hline 1010 & & 80 & 12 & 9.525 & $3 / 8$ & 55.475 & 2.1841 & 16610 & 3734 & 23291 & 5236 \\
\hline 210 & & 90 & 10 & 12.7 & $1 / 2$ & 57.30 & 2.2559 & 23162 & 5207 & 35083 & 7887 \\
\hline 310 & & 110 & 8 & 19.05 & $3 / 4$ & 60.95 & 2.3996 & 37828 & 8504 & 61835 & 13901 \\
\hline 1915 & 75 & 105 & 17 & 12.7 & $1 / 2$ & 80.289 & 3.1610 & 25008 & 5622 & 27895 & 6271 \\
\hline 215 & & 130 & 10 & 19.05 & $3 / 4$ & 82.550 & 3.2500 & 44762 & 10063 & 62119 & 13965 \\
\hline 1920 & 100 & 140 & 17 & 12.7 & $1 / 2$ & 105.969 & 4.172 & 44456 & 9994 & 46818 & 10525 \\
\hline 220 & & 180 & 10 & 25.4 & 1 & 111.531 & 4.3910 & 92639 & 20826 & 122161 & 27463 \\
\hline
\end{tabular}

Adapted from The Timken Company (Ref. 20). 
TABLE 2.-LIFE FACTORS FOR 30-MM-BORE DEEP-GROOVE AND ANGULAR-CONTACT BALL BEARINGS WITH ABEC-5 TOLERANCES

\begin{tabular}{|c|c|c|c|c|c|}
\hline \multirow{2}{*}{$\begin{array}{l}\text { ABMA } \\
\text { fit class }\end{array}$} & \multirow{2}{*}{$\begin{array}{c}\text { Clearance, } \\
\mathrm{mm}\end{array}$} & \multicolumn{2}{|c|}{ Inner-race Hertz stress } & \multirow{2}{*}{$\begin{array}{l}\text { Life factor fit } \\
\text { deep-groove }\end{array}$} & \multirow{2}{*}{$\begin{array}{l}\text { Life factor fit } \\
\text { angular contact }\end{array}$} \\
\hline & & $\mathrm{MPa}$ & ksi & & \\
\hline \multirow[t]{3}{*}{ j5-min } & +0.004 & 1200 & 175 & 1 & 1 \\
\hline & & 1720 & 250 & 1 & 1 \\
\hline & & 2240 & 325 & 1 & 1 \\
\hline \multirow[t]{3}{*}{$\mathrm{j} 5$} & -0.0035 & 1200 & 175 & 1 & 1 \\
\hline & & 1720 & 250 & 1 & 1 \\
\hline & & 2240 & 325 & 1 & 1 \\
\hline \multirow[t]{3}{*}{ j5-max } & -0.011 & 1200 & 175 & 0.74 & 0.82 \\
\hline & & 1720 & 250 & .81 & .87 \\
\hline & & 2240 & 325 & .85 & .90 \\
\hline \multirow[t]{3}{*}{ j6-min } & +0.004 & 1200 & 175 & 1 & 1 \\
\hline & & 1720 & 250 & 1 & 1 \\
\hline & & 2240 & 325 & 1 & 1 \\
\hline \multirow[t]{3}{*}{ j6 } & -0.0055 & 1200 & 175 & 0.94 & 0.96 \\
\hline & & 1720 & 250 & .96 & .97 \\
\hline & & 2240 & 325 & .97 & .98 \\
\hline \multirow[t]{3}{*}{ j6-max } & -0.015 & 1200 & 175 & 0.62 & 0.72 \\
\hline & & 1720 & 250 & .72 & .80 \\
\hline & & 2240 & 325 & .77 & .84 \\
\hline \multirow[t]{3}{*}{ k5-min } & -0.002 & 1200 & 175 & 1 & 1 \\
\hline & & 1720 & 250 & 1 & 1 \\
\hline & & 2240 & 325 & 1 & 1 \\
\hline \multirow[t]{3}{*}{$\mathrm{k} 5$} & -0.0095 & 1200 & 175 & 0.79 & 0.85 \\
\hline & & 1720 & 250 & .85 & .90 \\
\hline & & 2240 & 325 & .88 & .92 \\
\hline \multirow[t]{3}{*}{ k5-max } & -0.017 & 1200 & 175 & 0.56 & 0.67 \\
\hline & & 1720 & 250 & .67 & .76 \\
\hline & & 2240 & 325 & .74 & .82 \\
\hline \multirow[t]{3}{*}{$\mathrm{m} 5-\mathrm{min}$} & -0.008 & 1200 & 175 & 0.84 & 0.89 \\
\hline & & 1720 & 250 & .89 & .93 \\
\hline & & 2240 & 325 & .91 & .94 \\
\hline \multirow[t]{3}{*}{ m5 } & -0.0155 & 1200 & 175 & 0.60 & 0.70 \\
\hline & & 1720 & 250 & .70 & .79 \\
\hline & & 2240 & 325 & .76 & .84 \\
\hline \multirow[t]{3}{*}{ m5-max } & -0.023 & 1200 & 175 & 0.43 & 0.54 \\
\hline & & 1720 & 250 & .55 & .66 \\
\hline & & 2240 & 325 & .64 & .73 \\
\hline
\end{tabular}

Results averaged from size 1906 and 206 bearings. 
TABLE 3.-LIFE FACTORS FOR 50-MM-BORE DEEP-GROOVE AND ANGULAR-CONTACT BALL BEARINGS WITH ABEC-5 TOLERANCES

\begin{tabular}{|c|c|c|c|c|c|}
\hline \multirow{2}{*}{$\begin{array}{l}\text { ABMA } \\
\text { fit class }\end{array}$} & \multirow{2}{*}{$\begin{array}{c}\text { Clearance, } \\
\mathrm{mm}\end{array}$} & \multicolumn{2}{|c|}{ Inner-race Hertz stress } & \multirow{2}{*}{$\begin{array}{c}\text { Life factor } \\
\text { deep-groove }\end{array}$} & \multirow{2}{*}{$\begin{array}{c}\text { Life factor } \\
\text { angular-contact }\end{array}$} \\
\hline & & $\mathrm{MPa}$ & $\mathrm{ksi}$ & & \\
\hline \multirow[t]{3}{*}{ j5-min } & +0.005 & 1200 & 175 & 1 & 1 \\
\hline & & 1720 & 250 & 1 & 1 \\
\hline & & 2240 & 325 & 1 & 1 \\
\hline \multirow[t]{3}{*}{ j5 } & -0.0045 & 1200 & 175 & 0.99 & 0.99 \\
\hline & & 1720 & 250 & .99 & .99 \\
\hline & & 2240 & 325 & .99 & 1 \\
\hline \multirow[t]{3}{*}{ j5-max } & -0.014 & 1200 & 175 & 0.76 & 0.83 \\
\hline & & 1720 & 250 & .83 & .88 \\
\hline & & 2240 & 325 & .86 & .91 \\
\hline \multirow[t]{3}{*}{ j6-min } & +0.005 & 1200 & 175 & 1 & 1 \\
\hline & & 1720 & 250 & 1 & 1 \\
\hline & & 2240 & 325 & 1 & 1 \\
\hline \multirow[t]{3}{*}{ j6 } & -0.007 & 1200 & 175 & 0.92 & 0.95 \\
\hline & & 1720 & 250 & .95 & .96 \\
\hline & & 2240 & 325 & .96 & .97 \\
\hline \multirow[t]{3}{*}{ j6-max } & -0.019 & 1200 & 175 & 0.66 & 0.75 \\
\hline & & 1720 & 250 & .75 & .83 \\
\hline & & 2240 & 325 & .80 & .87 \\
\hline \multirow[t]{3}{*}{ k5-min } & -0.002 & 1200 & 175 & 1 & 1 \\
\hline & & 1720 & 250 & 1 & 1 \\
\hline & & 2240 & 325 & 1 & 1 \\
\hline \multirow[t]{3}{*}{ k5 } & -0.0115 & 1200 & 175 & 0.82 & 0.87 \\
\hline & & 1720 & 250 & .87 & .91 \\
\hline & & 2240 & 325 & .90 & .93 \\
\hline \multirow[t]{3}{*}{ k5-max } & -0.021 & 1200 & 175 & 0.62 & 0.72 \\
\hline & & 1720 & 250 & .72 & .80 \\
\hline & & 2240 & 325 & .78 & .85 \\
\hline \multirow[t]{3}{*}{$\mathrm{m} 5-\mathrm{min}$} & -0.009 & 1200 & 175 & 0.87 & 0.92 \\
\hline & & 1720 & 250 & .91 & .94 \\
\hline & & 2240 & 325 & .93 & .96 \\
\hline \multirow[t]{3}{*}{$\mathrm{m} 5$} & -0.0185 & 1200 & 175 & 0.67 & 0.76 \\
\hline & & 1720 & 250 & .76 & .83 \\
\hline & & 2240 & 325 & .81 & .87 \\
\hline \multirow[t]{3}{*}{ m5-max } & -0.028 & 1200 & 175 & 0.51 & 0.62 \\
\hline & & 1720 & 250 & .63 & .73 \\
\hline & & 2240 & 325 & .70 & .79 \\
\hline \multirow[t]{3}{*}{ m6-min } & -.009 & 1200 & 175 & 0.87 & 0.92 \\
\hline & & 1720 & 250 & .91 & .94 \\
\hline & & 2240 & 325 & .93 & .96 \\
\hline \multirow[t]{3}{*}{$\mathrm{m} 6$} & -0.021 & 1200 & 175 & 0.62 & 0.72 \\
\hline & & 1720 & 250 & .72 & .80 \\
\hline & & 2240 & 325 & .78 & .85 \\
\hline \multirow[t]{3}{*}{ m6-max } & -0.033 & 1200 & 175 & 0.44 & 0.55 \\
\hline & & 1720 & 250 & .57 & .67 \\
\hline & & 2240 & 325 & .65 & .74 \\
\hline
\end{tabular}

Results averaged from size 1910, 1010, 210, and 310 bearings. 
TABLE 4-LIFE FACTORS FOR 75-MM-BORE DEEP-GROOVE AND ANGULAR-CONTACT BALL BEARINGS WITH ABEC-5 TOLERANCES

\begin{tabular}{|c|c|c|c|c|c|}
\hline \multirow{2}{*}{$\begin{array}{l}\text { ABMA } \\
\text { fit class }\end{array}$} & \multirow{2}{*}{$\begin{array}{c}\text { Clearance, } \\
\mathrm{mm}\end{array}$} & \multicolumn{2}{|c|}{ Inner-race Hertz stress } & \multirow{2}{*}{$\begin{array}{c}\text { Life factor } \\
\text { deep-groove }\end{array}$} & \multirow{2}{*}{$\begin{array}{c}\text { Life factor } \\
\text { angular-contact }\end{array}$} \\
\hline & & $\mathrm{MPa}$ & ksi & & \\
\hline \multirow[t]{3}{*}{ j5-min } & +0.007 & 1200 & 175 & 1 & 1 \\
\hline & & 1720 & 250 & 1 & 1 \\
\hline & & 2240 & 325 & 1 & 1 \\
\hline \multirow[t]{3}{*}{$\mathrm{j} 5$} & -0.004 & 1200 & 175 & 1 & 1 \\
\hline & & 1720 & 250 & 1 & 1 \\
\hline & & 2240 & 325 & 1 & 1 \\
\hline \multirow[t]{3}{*}{ j5-max } & -0.015 & 1200 & 175 & 0.80 & 0.86 \\
\hline & & 1720 & 250 & .86 & .91 \\
\hline & & 2240 & 325 & .89 & .93 \\
\hline \multirow[t]{3}{*}{ j6-min } & +0.007 & 1200 & 175 & 1 & 1 \\
\hline & & 1720 & 250 & 1 & 1 \\
\hline & & 2240 & 325 & 1 & 1 \\
\hline \multirow[t]{3}{*}{ j6 } & -0.007 & 1200 & 175 & 0.94 & 0.96 \\
\hline & & 1720 & 250 & .96 & .97 \\
\hline & & 2240 & 325 & .97 & .98 \\
\hline \multirow[t]{3}{*}{ j6-max } & -0.021 & 1200 & 175 & 0.71 & 0.79 \\
\hline & & 1720 & 250 & .79 & .85 \\
\hline & & 2240 & 325 & .83 & .89 \\
\hline \multirow[t]{3}{*}{$\mathrm{k} 5-\min$} & -0.002 & 1200 & 175 & 1 & 1 \\
\hline & & 1720 & 250 & 1 & 1 \\
\hline & & 2240 & 325 & 1 & 1 \\
\hline \multirow[t]{3}{*}{$\mathrm{k} 5$} & -0.013 & 1200 & 175 & 0.84 & 0.89 \\
\hline & & 1720 & 250 & .88 & .92 \\
\hline & & 2240 & 325 & .91 & .94 \\
\hline \multirow{3}{*}{ k5-max } & -0.024 & 1200 & 175 & 0.66 & 0.76 \\
\hline & & 1720 & 250 & .75 & .83 \\
\hline & & 2240 & 325 & .80 & .87 \\
\hline \multirow[t]{3}{*}{ m5-min } & -0.011 & 1200 & 175 & 0.87 & 0.91 \\
\hline & & 1720 & 250 & .91 & .94 \\
\hline & & 2240 & 325 & .93 & .95 \\
\hline \multirow[t]{3}{*}{$\mathrm{m} 5$} & -0.0122 & 1200 & 175 & 0.69 & 0.78 \\
\hline & & 1720 & 250 & .78 & .85 \\
\hline & & 2240 & 325 & .82 & .88 \\
\hline \multirow[t]{3}{*}{ m5-max } & -0.033 & 1200 & 175 & 0.55 & 0.66 \\
\hline & & 1720 & 250 & .66 & .75 \\
\hline & & 2240 & 325 & .73 & .81 \\
\hline \multirow[t]{3}{*}{ m6-min } & -0.011 & 1200 & 175 & 0.87 & 0.91 \\
\hline & & 1720 & 250 & .91 & .94 \\
\hline & & 2240 & 325 & .93 & .95 \\
\hline $\mathrm{m} 6$ & -0.025 & 1200 & 175 & 0.65 & 0.75 \\
\hline & & 1720 & 250 & .74 & .82 \\
\hline & & 2240 & 325 & .80 & .86 \\
\hline m6-max & -0.039 & 1200 & 175 & 0.48 & 0.59 \\
\hline & & 1720 & 250 & .60 & .71 \\
\hline & & 2240 & 325 & .68 & .77 \\
\hline n6-min & -0.020 & 1200 & 175 & 0.72 & 0.80 \\
\hline & & 1720 & 250 & .80 & .86 \\
\hline & & 2240 & 325 & .84 & .89 \\
\hline $\mathrm{n} 6$ & -0.034 & 1200 & 175 & 0.54 & 0.65 \\
\hline & & 1720 & 250 & .65 & .75 \\
\hline & & 2240 & 325 & .72 & .80 \\
\hline n6-max & -0.048 & 1200 & 175 & 0.40 & 0.51 \\
\hline & & 1720 & 250 & .53 & .64 \\
\hline & & 2240 & 325 & .61 & .72 \\
\hline
\end{tabular}

Results averaged from size 1915 and 215 bearings. 
TABLE 5.-LIFE FACTORS FOR 100-MM-BORE DEEP-GROOVE AND ANGULAR-CONTACT BALL BEARINGS WITH ABEC-5 TOLERANCES

\begin{tabular}{|c|c|c|c|c|c|}
\hline \multirow{2}{*}{$\begin{array}{l}\text { ABMA } \\
\text { fit class }\end{array}$} & \multirow{2}{*}{$\begin{array}{c}\text { Clearance, } \\
\mathrm{mm}\end{array}$} & \multicolumn{2}{|c|}{ Inner-race Hertz stress } & \multirow{2}{*}{$\begin{array}{c}\text { Life factor } \\
\text { deep-groove }\end{array}$} & \multirow{2}{*}{$\begin{array}{c}\text { Life factor } \\
\text { angular-contact }\end{array}$} \\
\hline & & $\mathrm{MPa}$ & ksi & & \\
\hline \multirow[t]{3}{*}{$\mathrm{j} 5-\min$} & \multirow[t]{3}{*}{+0.009} & 1200 & 175 & 1 & 1 \\
\hline & & 1720 & 250 & 1 & 1 \\
\hline & & 2240 & 325 & 1 & 1 \\
\hline \multirow[t]{3}{*}{$\mathrm{j} 5$} & \multirow[t]{3}{*}{-0.0035} & 1200 & 175 & 1 & 1 \\
\hline & & 1720 & 250 & 1 & 1 \\
\hline & & 2240 & 325 & 1 & 1 \\
\hline \multirow[t]{3}{*}{ j5-max } & \multirow[t]{3}{*}{-0.016} & 1200 & 175 & 0.84 & 0.89 \\
\hline & & 1720 & 250 & .89 & .92 \\
\hline & & 2240 & 325 & .91 & .94 \\
\hline \multirow[t]{3}{*}{ j6-min } & \multirow[t]{3}{*}{+0.009} & 1200 & 175 & 1 & 1 \\
\hline & & 1720 & 250 & 1 & 1 \\
\hline & & 2240 & 325 & 1 & 1 \\
\hline \multirow[t]{3}{*}{ j6 } & -0.007 & 1200 & 175 & 0.96 & 0.97 \\
\hline & & 1720 & 250 & .97 & .98 \\
\hline & & 2240 & 325 & .98 & .99 \\
\hline j6-max & -0.023 & 1200 & 175 & 0.76 & 0.83 \\
\hline & & 1720 & 250 & .83 & .88 \\
\hline & & 2240 & 325 & .87 & .91 \\
\hline $\mathrm{k} 5$-min & -0.003 & 1200 & 175 & 1 & 1 \\
\hline & & 1720 & 250 & 1 & 1 \\
\hline & & 2240 & 325 & 1 & 1 \\
\hline $\mathrm{k} 5$ & -0.0155 & 1200 & 175 & 0.85 & 0.90 \\
\hline & & 1720 & 250 & .89 & .93 \\
\hline & & 2240 & 325 & .92 & .94 \\
\hline k5-max & -0.028 & 1200 & 175 & 0.71 & 0.78 \\
\hline & & 1720 & 250 & .79 & .85 \\
\hline & & 2240 & 325 & .83 & .88 \\
\hline $\mathrm{m} 5$-min & -0.013 & 1200 & 175 & 0.88 & 0.92 \\
\hline & & 1720 & 250 & .92 & .94 \\
\hline & & 2240 & 325 & .93 & .96 \\
\hline $\mathrm{m} 5$ & -0.0255 & 1200 & 175 & 0.73 & 0.81 \\
\hline & & 1720 & 250 & .81 & .86 \\
\hline & & 2240 & 325 & .85 & .90 \\
\hline m5-max & -0.038 & 1200 & 175 & 0.61 & 0.70 \\
\hline & & 1720 & 250 & .71 & .79 \\
\hline & & 2240 & 325 & .77 & .84 \\
\hline m6-min & -.013 & 1200 & 175 & 0.88 & 0.92 \\
\hline & & 1720 & 250 & .92 & .94 \\
\hline & & 2240 & 325 & .93 & .96 \\
\hline $\mathrm{m} 6$ & -0.029 & 1200 & 175 & 0.70 & 0.78 \\
\hline & & 1720 & 250 & .78 & .84 \\
\hline & & 2240 & 325 & .83 & .88 \\
\hline m6-max & -0.045 & 1200 & 175 & 0.55 & 0.64 \\
\hline & & 1720 & 250 & .66 & .74 \\
\hline & & 2240 & 325 & .73 & .80 \\
\hline n6-min & -0.023 & 1200 & 175 & 0.76 & 0.83 \\
\hline & & 1720 & 250 & .83 & .88 \\
\hline & & 2240 & 325 & .87 & .91 \\
\hline n6 & -0.039 & 1200 & 175 & 0.60 & 0.69 \\
\hline & & 1720 & 250 & .70 & .78 \\
\hline & & 2240 & 325 & .76 & .83 \\
\hline n6-max & -0.055 & 1200 & 175 & .47 & 0.57 \\
\hline & & 1720 & 250 & .59 & .69 \\
\hline & & 2240 & 325 & .67 & .76 \\
\hline P6-min & -0.037 & 1200 & 175 & 0.62 & 0.71 \\
\hline & & 1720 & 250 & .72 & .79 \\
\hline & & 2240 & 325 & .78 & .84 \\
\hline p6 & -0.053 & 1200 & 175 & 0.48 & 0.58 \\
\hline & & 1720 & 250 & .61 & .70 \\
\hline & & 2240 & 325 & .68 & .76 \\
\hline p6-max & -0.069 & 1200 & 175 & 0.38 & 0.47 \\
\hline & & 1720 & 250 & .51 & .61 \\
\hline & & 2240 & 325 & .60 & .69 \\
\hline
\end{tabular}

Results averaged from size 1920 and 220 bearings. 
The loading rollers were cylindrical (no crown) and presumably made from the equivalent of much harder American Iron and Steel Institute (AISI) 52100 steel. The surface speed was $14.25 \mathrm{~m} / \mathrm{s}$, which corresponds to $495 \mathrm{rpm}$ or $27225 \mathrm{DN}$ (low speed). The lubricant was "highly refined pure paraffinic oil" (Czyzewski (Ref. 3)).

Czyzewski (Ref. 3) reported inner-race life data in the form of Weibull plots for three test series: three levels of interference fits, 80, 40, and $5 \mathrm{MPa}(11.6,5.8$, and $0.725 \mathrm{ksi})$, given in terms of the bore-shaft interface pressure. The inner-race lives scaled from Czyzewski's plot are shown in Table 6 and are replotted and reanalyzed in Figure 9. The three test series had $L_{10}$ lives of $3.80 \times 10^{4}, 3.07 \times 10^{5}$, and $3.02 \times 10^{6}$ cycles and $L_{50}$ lives of $1.54 \times 10^{5}, 1.78 \times 10^{6}$, and $9.44 \times 10^{6}$ cycles, respectively.

Czyzewski reported (Ref. 3) that all of the races of series 1 burst (failed by fracture), three of ten races in series 2 fractured, and none of series 3 fractured. We suspect that incipient rollingelement fatigue spalls initiated these race fractures. The Czyzewski results suggest that there is a limit pressure above which inner ring fracture can occur.

Czyzewski's interference fits (Ref. 3) are extremely tight, particularly for series 1 . The Czyzewski bearing inner races are approximately the same size as those on the 210-size deepgroove ball bearing considered in this paper, for which the heaviest fit recommended is an $\mathrm{m} 6$ fit. At the tight end of the tolerance band, an $\mathrm{m} 6$ fit in this bearing produces an interface pressure of $16.7 \mathrm{MPa}(2.43 \mathrm{ksi})$. The Czyzewski series-1 fit pressure $(80 \mathrm{MPa})$ is 4.8 times higher than the pressure would be for the maximum $\mathrm{m} 6$ fit for this ball bearing.

Czyzewski's experiments (Ref. 3) may also be compared with results from 210-size roller bearings in Oswald et al.

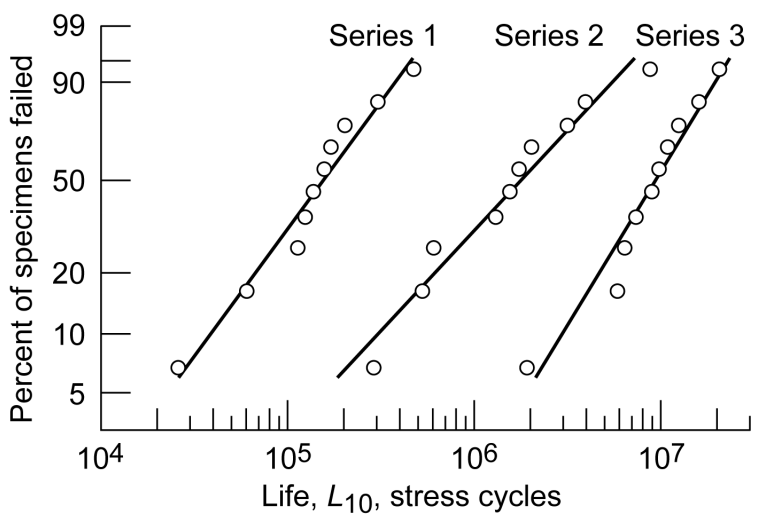

Figure 9.-Reanalyzed rolling contact test data from Czyzewski (Ref. 3). The three series had interface pressures of 80,40 , and $5 \mathrm{MPa}$ and $L_{10}$ lives of $3.80 \times 10^{4}, 3.07 \times 10^{5}$, and $3.02 \times 10^{6}$ cycles, respectively.

(Ref. 10), where at the tight end of the tolerance band, an m6 fit produces an interface pressure of $14.8 \mathrm{MPa}(2.15 \mathrm{ksi})$. The Czyzewski series-1 fit pressure is 5.4 times higher than the pressure would be for the tight end of the m6 fit for this roller bearing.

Czyzewski (Ref. 3) did not mention the radial load he employed for his tests or the resulting Hertz stress. He reported instead the maximum subsurface shear stress (including the effect of hoop stress) normalized to the maximum Hertz stress as values of $0.520,0.406,0.316$, and 0.302 for interference pressures of $80,40,5$, and $0 \mathrm{MPa}$ respectively.

TABLE 6-ROLLING-ELEMENT FATIGUE LIFE IN LOAD CYCLES FROM CZYZEWSKI (REF. 3) FOR THREE LEVELS OF ROLLER BEARING INTERFERENCE FIT PRESSURE: 80, 40 AND 5 MPA (11.6, 5.8 AND 0.725 ksi) FOR TEST SERIES 1, 2 AND 3, RESPECTIVELY

\begin{tabular}{|l|c|c|c|}
\hline \multicolumn{1}{|c|}{ Point } & Series 1 & Series 2 & Series 3 \\
\hline Max shear stress, MPa (ksi) & $390(56.6)$ & $305(44.2)$ & $237(34.4)$ \\
$\mathrm{L}_{10}$ life, cycles & $3.80 \times 10^{4}$ & $3.07 \times 10^{5}$ & $3.02 \times 10^{6}$ \\
$\mathrm{~L}_{50}$ life, cycles & $1.54 \times 10^{5}$ & $1.78 \times 10^{6}$ & $9.44 \times 10^{6}$ \\
Weibull slope, $e$ & 1.35 & 1.07 & 1.65 \\
Failure index & $10 / 10$ & $10 / 10$ & $10 / 10$ \\
\hline 1 & ${ }^{\mathrm{a}} 25620$ & ${ }^{\mathrm{a}} 290000$ & 1915000 \\
2 & ${ }^{\mathrm{a}} 60000$ & 530000 & 5910000 \\
3 & ${ }^{\mathrm{a}} 112800$ & ${ }^{\mathrm{a}} 610000$ & 6460000 \\
4 & ${ }^{\mathrm{a}} 123800$ & ${ }^{\mathrm{a}} 1300000$ & 7380000 \\
5 & ${ }^{\mathrm{a}} 136700$ & 1550000 & 9000000 \\
6 & ${ }^{\mathrm{a}} 156000$ & 1740000 & 9800000 \\
7 & ${ }^{\mathrm{a}} 169900$ & 2040000 & 10900000 \\
8 & ${ }^{\mathrm{a}} 203000$ & 3160000 & 12500000 \\
9 & ${ }^{\mathrm{a}} 304000$ & 3950000 & 16100000 \\
10 & ${ }^{\mathrm{a}} 476000$ & 8800000 & 20900000 \\
\hline
\end{tabular}

${ }^{\mathrm{a}}$ Race fractured at failure. 


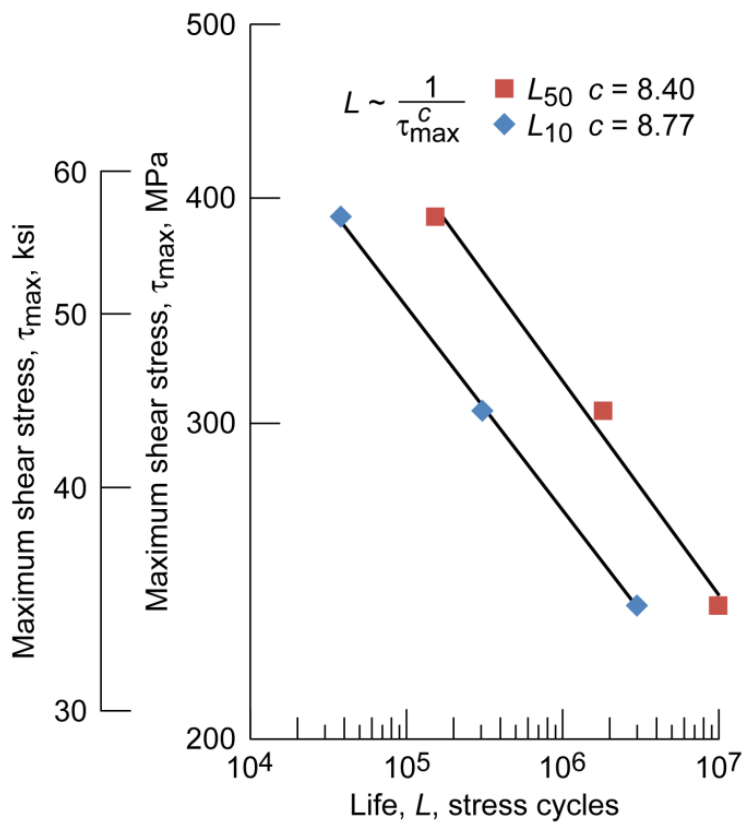

Figure 10.-Relationship between life and maximum subsurface shear stress for Czyzewski's rollingelement fatigue tests (Ref. 3) showing the relationship between life and the shear stress-life exponent, $c$.

From the given data, we calculated the hoop stress at $z$ (the depth below the surface to the maximum shearing stress) to be 325,162 , and 20.3 $\mathrm{MPa}(47,24$, and $2.9 \mathrm{ksi})$ for the three test series, respectively. From the hoop stress and the maximum shear stress, we estimated the maximum Hertz stress to be approximately $750 \mathrm{MPa}(110 \mathrm{ksi})$. This is a very low stress value; however, the test inner race is made from annealed material that will have a much shorter life than rings made from typical Rockwell C 60 hardness material.

From our estimate of $750 \mathrm{MPa}(110 \mathrm{ksi})$ maximum Hertz stress and from Czyzewski's reported normalized maximum subsurface shear stress, we calculated the actual maximum subsurface shear stress to be 390,305 , and $237 \mathrm{MPa}(56.6,44.2$, and $34.4 \mathrm{ksi}$ ) for series 1,2 , and 3, respectively. We calculated the subsurface shear stress with no interference fit to be 227 MPa (33 ksi).

In Figure 10, we show the relationship between the normalized maximum subsurface shear stress and the inner-race life for Czyzewski's data. The shear stress-life exponent $c$ was found to be 8.77 for the $L_{10}$ life and 8.40 for the $L_{50}$ life. The $L_{10}$ result compares reasonably well with the shear stress-life exponent $c$ of 9 assumed by Coe and Zaretsky (Ref. 4) and by Oswald et al. (Ref. 10).

Using a power-series curve fit, we calculated the $L_{10}$ and $L_{50}$ lives for no interference fit to be $4.24 \times 10^{6}$ and $1.73 \times 10^{7}$ cycles. This suggests that inner-race $L_{10}$ fatigue lives were reduced by 99, 93, and 29 percent and that the $L_{50}$ fatigue lives were reduced by 99,90 , and 45 percent by the interference fits of series 1,2 , and 3 , respectively.
The series 3 fit, which reduced the race $L_{10}$ life by 29 percent and which had an interface pressure of $5 \mathrm{MPa}(0.725 \mathrm{ksi})$, is comparable to the pressure from the tight end of a j5 fit on a 210 -size roller bearing $(5.1 \mathrm{MPa}(0.74 \mathrm{ksi})$, which produces a life reduction of 20 percent. The other two Czyzewski fits (Ref. 3 ) are much tighter than any of the recommended interference fits in ANSI/ABMA-7 (Ref. 1).

It is commonly accepted that where tight interference fits are used and/or large hoop stresses are induced in bearing inner rings, case carburizing should be utilized to prevent ring fracture from incipient rolling-element fatigue spalls. The typical explanation is that the soft ductile core of the case-carburized steel prevents crack propagation through the core. However, in the Czyzewski experiments (Ref. 3), the ring hardness is less than Rockwell C 15, which is significantly lower than the core of case-carburized steels such as M-50 NiL and AISI 9310 (Zaretsky (Ref. 7)). We theorize that it is the compressive residual stresses induced by heat treatment in case-carburized steels rather than the ductile (soft) core that offsets the effect of hoop stresses to prevent ring fracture.

\section{Results and Discussion}

The analysis described in the previous sections was applied to radially loaded deep-groove ball bearings and to thrustloaded angular-contact ball bearings made of AISI 52100 steel. Table 1 lists the dimensions, static and dynamic load capacity of the bearings. Identical dimensions were chosen for the two bearing types except for the contact angle: $0^{\circ}$ for the deepgroove bearings and $25^{\circ}$ for the angular-contact bearings.

The analysis included four bore sizes and either two or four dimension series for each bore size. The dimension series are shown schematically in Figure 11. Each bearing was analyzed at three levels of inner-race Hertz stress. A commercial bearing analysis code (Poplawski et al. (Ref. 24)) was used to calculate the unfactored $L_{10}$ lives for the inner and outer races operating without interference fit. Life factors were calculated for up to seven fit classes for each bearing, with each fit taken at the maximum, average, and minimum values within the fit class for ABEC-5 tolerances (ANSI/ABMA-20 (Ref. 2)). (ABEC, Annular Bearing Engineering Committee of the American Bearing Manufacturers Association, is a system for specifying bearing tolerances with an odd number between 1 and 9 . ABEC-5 is a medium tolerance level.) Figure 12 shows a graphical representation of the shaft fits (adapted from ANSI/ABMA-7 (Ref. 1)). Harris (Ref. 26) discusses the effect of surface finish on interference fit as a result of the smoothing of asperities on the surface. He recommends reducing the calculated interference to account for asperity smoothing, depending on the quality of the finish. For very accurately ground surfaces, the reduction is $4 \mu \mathrm{m}$ ( $2 \mu \mathrm{m}$ for each surface: the inside diameter of the bore and the outside diameter of the shaft). In this work, the apparent interference was reduced by $4 \mu \mathrm{m}\left(160 \times 10^{-6}\right.$ in.) to account for surface finish effects. 


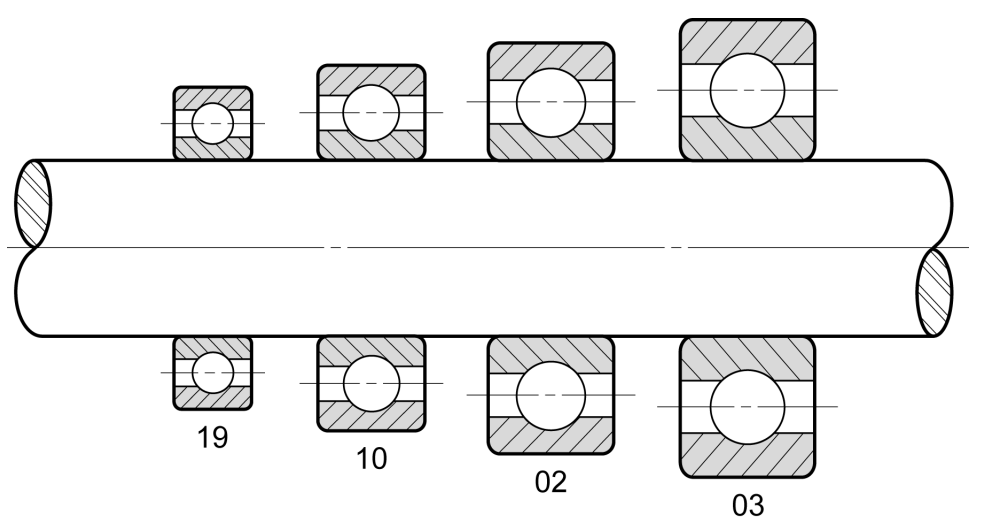

Figure 11.-ANSI/ABMA ball bearing dimension series (adapted from The Timken Company (Ref. 20)).

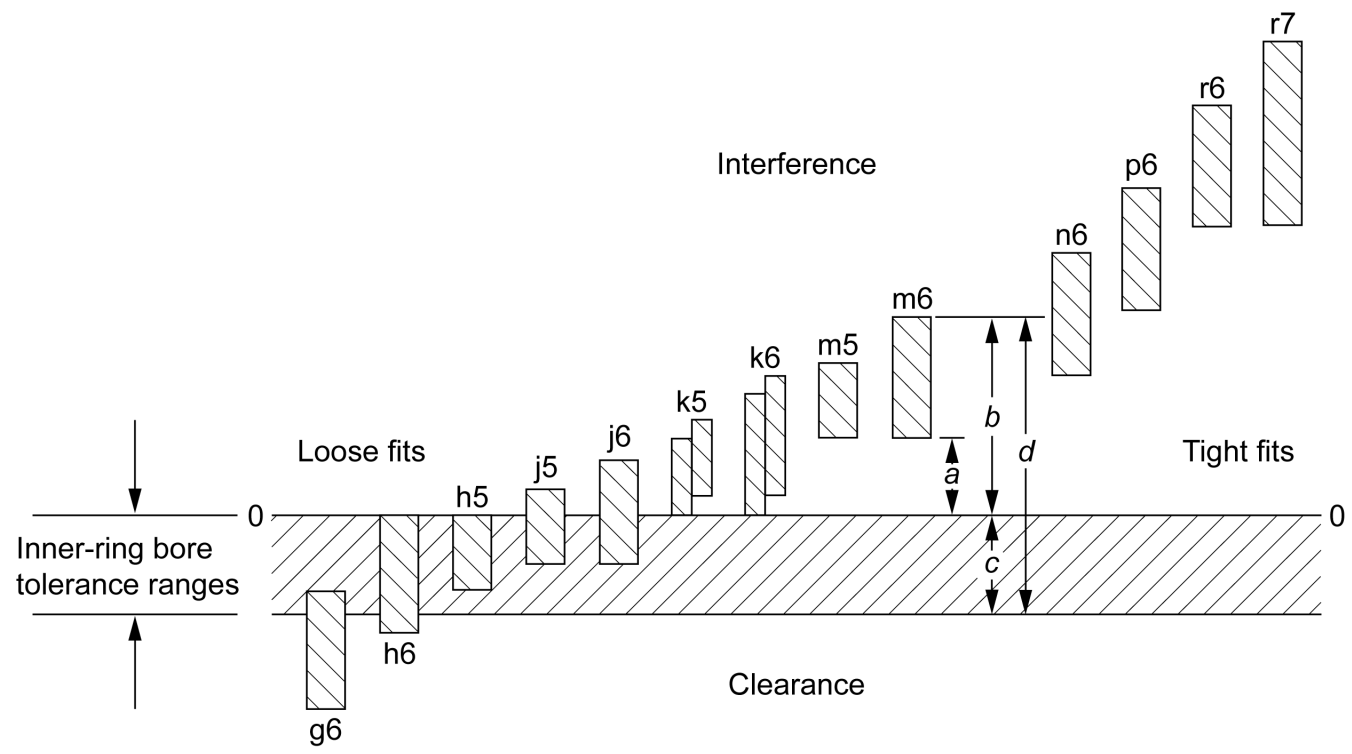

Figure 12.-Graphical representation of ANSI/ABMA shaft fits (adapted from ABMA (Ref. 1)). Dimensions illustrate $\mathrm{m} 6$ fit, with minimum fit within tolerance band represented by dimension $a$, maximum fit by dimension $d$. Fit classes $\mathrm{j} 5$ to $\mathrm{p} 6$ were considered in this paper.

Bearing dynamic load ratings were developed by Lundberg and Palmgren based on a geometry and material coefficient designated $f_{c}$ (currently called $f_{c m}$ in the standards (ANSI/ABMA-9 (Ref. 22))) that was benchmarked to unreported experimental data (Lundberg and Palmgren (Ref. 6)). Unfortunately, the interference fits used to generate the test data were also not reported. We have calculated life factors based on the assumption that the Lundberg and Palmgren bearing data did not include the effect of interference fit.

For this article, all bearings were modeled at the zero internal operating clearance condition and low speed. In the case of inner-ring interference fit or thermal strain, this means that the bearings would have an appropriate initial (unmounted) clearance between the balls and the races. In addition, the analysis assumes that the bearing speed is less than one million DN. For high-speed bearings or unusual thermal environments, an appropriate analysis code should be used.

Over 1150 bearing configurations and load cases were analyzed in our study. Each bearing was analyzed for three values of inner-race maximum Hertz stress: 1200, 1720, and $2240 \mathrm{MPa}(175,250$, and $325 \mathrm{ksi})$. The load on each bearing was chosen to produce the desired stress value. These loads are designated light, normal, and heavy, respectively. The analysis code calculated the inner- and outer-race lives using the traditional Lundberg-Palmgren (Ref. 6) method. Therefore, these lives implicitly include the life of the ball set.

Equation (13) was used to find the ratio of the inner- and outer-race lives. The adjusted value of the inner-race life (with the ball set life separated) was calculated from Equation (14a) 
for deep-groove bearings and from Equation (14b) for angular-contact bearings.

Data for limiting values of bearing bore sizes were taken from the table for tolerance class ABEC-5 (ANSI/ABMA-20 (Ref. 2)). Because this reference does not have information for shaft size limits, values for shaft diameter deviations were taken from the ANSI/ABMA shaft-fitting practice table (ANSI/ABMA-7 (Ref. 1)), which gives tolerance limits for bearings with the ABEC-1 quality level. The practice of using bore tolerances from an ABEC-5 table and shaft tolerances from an $\mathrm{ABEC}-1$ table is consistent with an example given by Harris (Ref. 26).

The shaft diameter was subtracted from the bore diameter and then $0.004 \mathrm{~mm}\left(160 \times 10^{-6}\right.$ in. $)$ was added to the difference to account for asperity smoothing. If the resulting fit was positive (indicating clearance), the interface pressure and, thus, the hoop stress were assumed to be zero. If the fit was negative, the resulting interference (as a positive number) was used to calculate the interface pressure due to the chosen interference fit in Equation (5).

The simplified procedure described in the next section was used to find $\left(\tau_{\max }\right)_{h}$. Equation (15) was used to calculate the life ratio (hence the revised life) for the inner race, and finally, the life of the entire bearing was calculated from Equations (16a) or (16b) using the reduced life of the inner race and the original lives of the ball set and outer race.

Ball bearings have an elliptical (point) contact between the ball and the inner race rather than the rectangular contact of roller bearings. Therefore, ball bearings have an inverseellipticity ratio $b / a$ different from zero. (Elliptical contact dimensions $a$ and $b$ are illustrated in Fig. 2.) The bearings considered herein have inner-race conformity $f_{i}=0.52$. From the bearing analysis code, it was found that $b / a=0.11$, $\tau_{\max } / S_{\max }=0.317$, and $u=0.765$. Similar results can be obtained from Figure 4 (Jones (Ref. 5)) using $b / a=0.11$.

Curve fits were calculated for $\tau_{\max } / S_{\max }$ and $u$ in terms of $b / a$ from the data given in Figure 4 (Jones (Ref. 5)). These equations may be used to find parameters for the procedure in the following section to calculate the life factor for bearings with conformities different from 0.52 , where the value of $b / a$ differs from 0.11 .

$$
\begin{aligned}
\frac{\tau_{\max }}{S_{\max }} & =0.1002\left(\frac{b}{a}\right)^{3} \\
& -0.2431\left(\frac{b}{a}\right)^{2}+0.1639\left(\frac{b}{a}\right)+0.30 \\
u & =0.3108\left(\frac{b}{a}\right)^{3}-0.5131\left(\frac{b}{a}\right)^{2} \\
& -0.1159\left(\frac{b}{a}\right)+0.786
\end{aligned}
$$

The bearing analysis code was used to verify that the shear stress ratio and the depth to maximum shear given in Equations (21) and (22) is correct for all the bearings analyzed here. In addition, one case of angular-contact bearings was checked that had different conformities: 0.515 for the inner race and 0.53 for the outer race. For the inner race on these bearings, $b / a=0.139, \tau_{\max } / S_{\max }=0.320$, and $u=0.760$. These changes have no effect (within the precision reported here) on the calculation of the life factor. (Note, however, that the different conformities alter the lives of the races and that the lives affect the life ratio.)

\section{Analysis of 210-Size Deep-Groove Ball Bearing With m6 Fit}

As an example of the methods presented herein, consider a 210-size deep-groove ball bearing carrying a normal radial load of $1847 \mathrm{~N}$ (415.1 lbf) and a middle-of-the-tolerance-band m6 inner-ring interference fit. From the analysis code or Equation (20), $S_{\max }=1720 \mathrm{MPa}(250 \mathrm{ksi})$. The analysis code predicted the $L_{10}$ life of the bearing as 3054 million inner-race revolutions and the outer- and inner-race lives as 19950 and 3443 million revolutions:

From Equation (13), the ratio of the outer-race life to innerrace life is $X=19950 / 3443=5.79$. The adjusted life of the inner race was found by solving Equation (14a):

$$
L_{I R-a d j}=L_{10}\left(\frac{2}{X^{e}}+1\right)^{1 / e}
$$

The solution gives $L_{I R-a d j}=3827$ million revolutions. (Although not needed for this calculation, the adjusted life of the outer race is 22175 million revolutions.)

The ANSI/ABMA shaft-fitting practice table for ABEC-1 bearings (ANSI/ABMA-7 (Ref. 1)) was used to find limiting diameters for a 50-mm (2-in.) shaft with an m6 fit. Figure 12 illustrates the shaft fits schematically, showing deviations from the nominal bearing bore and shaft diameter of $50.000 \mathrm{~mm}$ (1.9685 in.). The shaft deviation can range from 0.009 to $0.025 \mathrm{~mm}$ (350 to $1000 \times 10^{-6}$ in.) (shown as dimensions $a$ and $b$ in the figure). The bearing bore deviations were found in the table for tolerance class ABEC-5 (ANSI/ABMA20 (Ref. 2)). The bore deviation can range from 0.000 to $0.008 \mathrm{~mm}\left(0\right.$ to $-300 \times 10^{-6}$ in.) (shown as 0 and dimension $c$ in Fig. 12.)

The loosest within-tolerance $\mathrm{m} 6$ interference fit $(0.009 \mathrm{~mm}$, or $350 \times 10^{-6}$ in., tight) occurs when the largest bore $(50 \mathrm{~mm})$ is mounted on the smallest shaft $(50.009 \mathrm{~mm}$ or $1.9689 \mathrm{in}$.) (before adjusting for surface finish), shown as dimension $a$ in Figure 12. The tightest within-tolerance fit $(0.033 \mathrm{~mm}$, or $1300 \times 10^{-6}$ in., tight) occurs when the smallest bore (49.992 $\mathrm{mm}$ or $1.9682 \mathrm{in}$.) is mounted on the largest shaft (50.025 $\mathrm{mm}$ or 1.9695 in.), shown as dimension $d$ in Figure 12. The average of these extremes is an interference fit of $0.021 \mathrm{~mm}\left(830 \times 10^{-6}\right.$ in. $)$ tight. This interference fit was 
reduced by $0.004 \mathrm{~mm}\left(160 \times 10^{-6} \mathrm{in}\right.$.) to account for asperity smoothing, assuming smooth-ground surfaces. The resulting middle-of-the-tolerance-band $\mathrm{m} 6$ interference fit was 0.017 $\mathrm{mm}\left(670 \times 10^{-6}\right.$ in. $)$ tight.

For the example bearing, $D_{S}=50 \mathrm{~mm}$ (1.9685 in.), $D_{I R}=57.3 \mathrm{~mm}$ (2.2559 in.), ball diameter $d=12.7 \mathrm{~mm}(0.500$ in.), race conformity $f_{i}=0.52$, bearing ring width $W=20 \mathrm{~mm}$ (0.7874 in.), $S_{\max }=1720 \mathrm{MPa}(250 \mathrm{ksi}), E=205878 \mathrm{MPa}$ $\left(29.86 \times 10^{3} \mathrm{ksi}\right)$, Poisson's ratio $v=0.3$, and diametrical interference $\Delta=0.017 \mathrm{~mm}\left(670 \times 10^{-6} \mathrm{in}\right.$. $)$.

The life factor for the inner race due to the interference-fit stress was calculated by the following simplified procedure (adapted from Zaretsky (Ref. 7)) to calculate the maximum shear stress including the effect of hoop stress:

(1) Determine the maximum shearing stress from Equation (4), where $\tau_{\max }=-(0.317) S_{\max }=-545.2 \mathrm{MPa}$ $(-79.08 \mathrm{ksi})$.

(2) Determine the effective outside diameter of the inner race from Equation (8a), where $D_{\text {eff }}=58.94 \mathrm{~mm}$ (2.3205 in.).

(3) Determine the contact pressure from Equation (5), $p_{i}=9.81 \mathrm{MPa}(1.423 \mathrm{ksi})$.

(4) Determine $R^{\prime}$, where $R^{\prime}=D_{I R} / d=4.5118$ (dimensionless).

(5) Determine $k_{3}$, where $k_{3}=E\left(R^{\prime}+1\right) /\left[4\left(1-v^{2}\right) S_{\max }\right]=$ 181.25 (dimensionless).

(6) Use the value for the nondimensional depth for an inner race conformity of $f_{i}=0.52: u=0.765$ (dimensionless).

(7) Calculate the diameter to the maximum shear stress: $D=D_{I R}\left[1-u / k_{3}\right]=57.058 \mathrm{~mm}(2.246 \mathrm{in}$.).

(8) Calculate the hoop stress at $D$ from Equation (9): $\sigma_{h}=52.065 \mathrm{MPa}(7.55 \mathrm{ksi})$.

(9) Substitute values for $\tau_{\max }$ and $\sigma_{h}$ in Equations (1a) or (1b) to calculate $\left(\tau_{\max }\right)_{h}=-571.27 \mathrm{MPa}(-82.86 \mathrm{ksi})$.

(10) Compute the life ratio for the inner race from Equation (15): $L R=[-545.2 /(-571.27)]^{9}=0.6572$.

The inner-race life ratio from step (10) was used in Equation (16a) to calculate the life of the bearing, including the effect of the interference fit. The result was 2169 million inner-race revolutions:

$$
\begin{aligned}
\frac{1}{(L)_{h}^{1.11}} & =\frac{1}{(0.6572 \cdot 3827)^{1.11}}+\frac{2}{(5.79 \cdot 3827)^{1.11}} \\
& =\frac{1}{2169^{1.11}}
\end{aligned}
$$

The life factor for hoop stress from Equation (17) is

$$
(L F)_{h}=\frac{2169}{3054}=0.71
$$

Therefore, the middle-of-the-tolerance-band $\mathrm{m} 6$ interference fit will reduce the life of this bearing by 29 percent.

\section{Analysis of 210-Size Angular-Contact Ball Bearing With m6 Fit}

Consider a thrust-loaded angular-contact ball bearing comparable to the radially loaded deep-groove example given in the previous section. For a thrust load of $4010 \mathrm{~N}(581.6 \mathrm{lbf})$, the analysis code gives $S_{\max }$ as $1720 \mathrm{MPa}(250 \mathrm{ksi})$. Note that the applied thrust load to produce the same $S_{\max }(1720 \mathrm{MPa})$ is much higher than the radial load in the example in the previous section. This is because all balls carry the maximum load and maximum stress.

The analysis code predicts the $L_{10}$ life of the bearing as 550.3 million inner-race revolutions and the outer- and innerrace lives as 3447 and 624.2 million revolutions. The ratio of the outer-race life to the inner-race life $X=3447 / 624.2=5.52$.

Equation (14b) was solved in Equation (26) to find the adjusted life of the inner race: 1097 million revolutions. The adjusted life of the outer race was 6057 million revolutions:

$$
L_{I R-a d j}=L_{10}\left(2+\frac{1}{X^{e}}\right)^{1 / e}
$$

As in the deep-groove bearing example in the previous section, a middle-of-the-tolerance-band $\mathrm{m} 6$ fit with smoothly ground surfaces was used. This produced an effective interference of $0.017 \mathrm{~mm}\left(670 \times 10^{-6} \mathrm{in}\right.$.).

For the example bearing, $D_{S}=50 \mathrm{~mm}(1.9685$ in.), $D_{I R}=57.3 \mathrm{~mm}(2.2559$ in. $), d=12.7 \mathrm{~mm}(0.500$ in. $)$, $f_{i}=0.52, W=20 \mathrm{~mm}\left(0.7874 \mathrm{in}\right.$.), $S_{\max }=1720 \mathrm{MPa}(250 \mathrm{ksi})$, $E=205878 \mathrm{MPa}\left(29.86 \times 10^{3} \mathrm{ksi}\right), v=0.3, \Delta=0.017 \mathrm{~mm}$ $\left(670 \times 10^{-6}\right.$ in.), and contact angle $\alpha=25^{\circ}$.

Next, the life factor of the inner race due to the added stress from the interference fit was calculated from the following simplified procedure (adapted from Zaretsky (Ref. 7)) to calculate the maximum shear stress including the effect of hoop stress:

(1) Determine the maximum shearing stress from Equation (4), where $k_{1}=0.317: \tau_{\max }=-545.24 \mathrm{MPa}(-79.08 \mathrm{ksi})$.

(2) Determine the effective outside diameter of the inner race from Equation (8b): $D_{\text {eff }}=58.12 \mathrm{~mm}$ (2.288 in.). (We assume that the inner race shoulder is relieved.)

(3) Determine the contact pressure from Equation (5): $p_{i}=9.10 \mathrm{MPa}(1.320 \mathrm{ksi})$.

(4) Determine $R^{\prime}$, where $R^{\prime}=D_{I R} / d=4.5118$ (dimensionless).

(5) Determine $k_{3}$, where $k_{3}=E\left(R^{\prime}+1\right) /\left[4\left(1-v^{2}\right) S_{\max }\right]=$ 181.25 (dimensionless).

(6) Use the value for the nondimensional depth for an innerrace conformity of $f_{i}=0.52: u=0.765$ (dimensionless).

(7) Compute the $z$-component of the nondimensional depth, $u_{z}=u \cos (\alpha)=0.693$.

(8) Calculate the diameter, $D$ to the maximum shear stress (note that a term was added because of the contact angle, see Fig. 13): $D=D_{I R}\left(1-u_{z} / k_{3}\right)+d / 2(1-\cos \alpha)=$ $57.696 \mathrm{~mm}(2.271 \mathrm{in}$.). 
(9) Calculate the hoop stress at $D$ from Equation (9): $\sigma_{h}=52.21 \mathrm{MPa}(7.57 \mathrm{ksi})$.

(10) Substitute values for $\tau_{\max }$ and $\sigma_{h}$ in Equations (1a) or (1b) to calculate $\left(\tau_{\max }\right)_{h}=-571.34 \mathrm{MPa}(-82.87 \mathrm{ksi})$.

(11) Compute the life ratio for the inner race from Equation (15): $L R=[-545.24 /(-571.34)]^{9}=0.6565$.

The inner-race life ratio from step (11) was used in Equation (16b) to calculate the life of the bearing, including the effect of the interference fit. The result was 441.5 million inner-race revolutions:

$$
\begin{aligned}
\frac{1}{(L)_{h}^{1.11}}= & \frac{1}{(0.6565 \cdot 1097)^{1.11}} \\
& +\frac{1}{1097^{1.11}}+\frac{1}{(5.52 \cdot 1097)^{1.11}}=\frac{1}{441.5^{1.11}}
\end{aligned}
$$

From Equation (17), the life factor for the entire angularcontact bearing for hoop stress is

$$
(L F)_{h}=\frac{441.5}{550.3}=0.80
$$

Therefore, the middle-of-the-tolerance-band $\mathrm{m} 6$ interference fit will reduce the life of this bearing by 20 percent.

If the correction for the contact position of the ball on the race in steps (7) to (8) is neglected, the calculated value of the inner-race life ratio will change by less than 0.5 percent. This makes no difference in the calculated life ratio of the bearing to within the two decimal digits shown.

In this analysis, it is assumed that the contact angle of the bearing will remain at $25^{\circ}$, regardless of the thrust load. This means that the unloaded contact angle must be less than $25^{\circ}$. For the normal-load example described, the unloaded contact angle would be $20.4^{\circ}$ to produce a loaded contact angle of $25^{\circ}$. With the smaller contact angle, the bearing life would be about

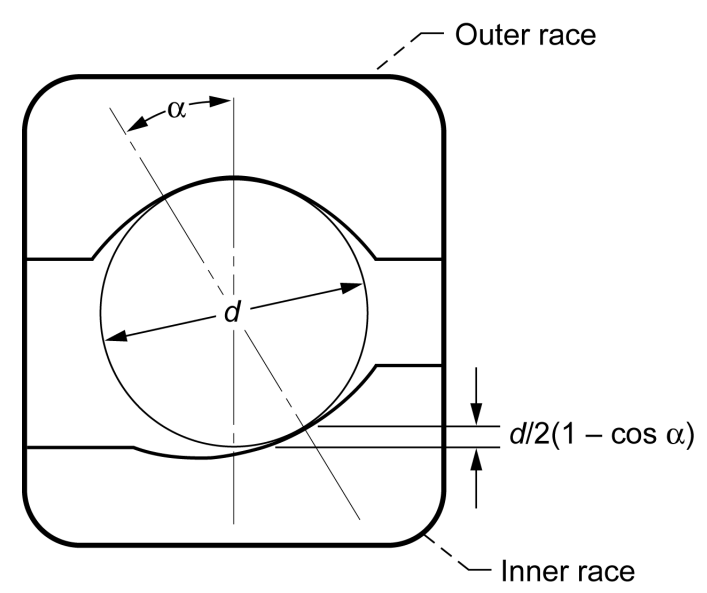

Figure 13.-Contact position on angular-contact bearing. $\alpha$, contact angle; $d$, diameter of ball.
1 percent smaller at the same inner-race maximum Hertz stress but there would be no difference in the calculated life factor.

\section{Interference-Fit Life Factors for ABEC-5 Ball Bearings}

The analysis described in the previous sections was applied to over 1150 configurations and load cases of deep-groove and angular-contact ball bearings. It included four bore sizes, up to four dimension series (extremely light, extra light, light, and medium), at three values of inner-race Hertz stress and up to seven inner-ring interference-fit classes. Each fit class was evaluated at the minimum, average, and maximum fit level for the ABEC -5 tolerance class.

The shaft interference table from ANSI/ABMA-7 (Ref. 1) (as illustrated in Fig. 12) shows fit classes ranging from g6 (loose) to $\mathrm{r} 7$ (heavy interference). There is no effect on life for loose fits that produce no pressure at the bore, and no values are given for very heavy fits on small bearings because these are not recommended. Hence, life factors were calculated for four fit classes (j5 to $\mathrm{m} 5$ ) for $30-\mathrm{mm}$ bearings (Table 2), five classes (j5 to $\mathrm{m} 6$ ) for 50 - $\mathrm{mm}$ bearings (Table 3), six classes (j5 to $\mathrm{n} 6$ ) for $75-\mathrm{mm}$ bearings (Table 4), and seven classes (j5 to p6)) for 100-mm bearings (Table 5). The k6 fit was not included in the analyses.

All interference fits were adjusted for the effect of asperity smoothing (assuming accurately ground surfaces) by adding $0.004 \mathrm{~mm}\left(160 \times 10^{-6}\right.$ in. $)$ to the clearance between the shaft and inner ring. If the resulting clearance value was negative (indicating interference), then the pressure and the resulting life factor were calculated.

The life factors were lower (greater life reduction) for deepgroove ball bearings than for thrust-loaded angular-contact ball bearings. However, the deep-groove bearings had longer lives for the same value of maximum Hertz stress. Note that, in a deep-groove bearing, the maximum stress occurs on only one ball at a time, but in an angular-contact ball bearing, all the balls carry the maximum load continuously.

The various bearing dimension series within a particular bore size exhibited almost identical results for the interference-fit life factor for a particular fit, despite significant differences in the interface pressure at the bore required to produce that fit. For example, the four 50-mm deep-groove bearings analyzed (sizes $1910,1010,210$, and 310) for an average $m 6$ interference fit of $0.021-\mathrm{mm}\left(827 \times 10^{-6} \mathrm{in}\right.$.) (before adjusting for finish effect) had fit pressures of $6.64,7.83,9.81$, and $13.13 \mathrm{MPa}(0.963,1.14$, 1.42 , and $1.90 \mathrm{ksi})$, respectively. However, the resulting life factors were nearly identical $(0.80,0.77,0.77$, and 0.78 for the heavy load case). Therefore, Tables 2 to 5 combine these results, showing the average life factors for each bore size.

Although the different dimension series within a particular bore size had similar life factors, the life factor was not the same for different bore sizes. In general, the larger bore sizes had a slightly lower life factor.

The life factors found in this study range from 1.00 (no effect) - where there was no net interface pressure - to a worst case 
of 0.38 (62-percent life reduction) for the maximum p6 fit on a 100 -mm-bore deep-groove ball bearing at $1200 \mathrm{MPa}$ (175 ksi) maximum Hertz stress. The corresponding angular-contact bearing had a life factor of 0.47 (53-percent life reduction) under the same conditions. However, the angular contact bearing, which carries a much higher load, had a much shorter life - about 9270 million revolutions for the 220 size, reduced to 4495 million revolutions by the maximum $\mathrm{p} 6$ interference fit, in contrast to the 220-size deep-groove bearing, which had a life of 51475 million revolutions, reduced to 18810 million revolutions.

As expected, tighter fits produced smaller life factors (i.e., shorter lives). In addition, the life factor was smallest (greatest life reduction) for bearings running under a light load, where the added stress due to the fit has a greater impact on the life.

Table 3 shows the variation (minimum to maximum) in life factor for 50-mm-bore bearings operating under three load cases (three levels of Hertz stress) at the five fit classes considered (j5 to m6). Figure 14 shows lives for the light and heavy load cases averaged from size 1910, 1010, 210, and 310 bearings (Table 3 data without the average fits). In the three loosest fit classes (j5 to $\mathrm{k} 5$ ), the minimum fit will produce no interface pressure; hence, the life factor is 1.00 .

For example, "k5-min" in the seventh row of Table 3 shows a "clearance" of $-0.002 \mathrm{~mm}\left(-79 \times 10^{-6}\right.$ in.). (This is actually a slight interference.) However, after adjusting for asperity smoothing, it becomes a clearance of $0.002 \mathrm{~mm}$. There is no interface pressure for a clearance; thus, $(L F)_{h}=1.0$. In the bar chart of Figure 14(a), the nonshaded part of the third bar that represents this $\mathrm{k} 5$-min fit shows an $L F$ of 1.0 .

For the heavier fits, the life factor is much smaller, especially at the light load level of $1200 \mathrm{MPa}(175 \mathrm{ksi})$ maximum Hertz stress, where life is higher. The smallest life factor found for 50 $\mathrm{mm}$ deep-groove bearings was 0.44 (56-percent life reduction). Likewise, on a 50-mm angular-contact bearing, the smallest life factor was 0.55 (45-percent life reduction). These values are represented by the shaded part of the right-most pair of bars in Figure 14(a).

On both bearings, the interference for "m6-max" was $0.033 \mathrm{~mm}$, reduced to $0.029 \mathrm{~mm}\left(1300 \times 10^{-6}\right.$ in.) by asperity smoothing. This fit produced an interface pressure of $22.40 \mathrm{MPa}$ (3.25 ksi) on the 310 -size deep-groove bearing and $21.00 \mathrm{MPa}$ (3.05 ksi) on the 310 -size angular-contact bearing. (Interface pressures are not shown in the tables.)

Figures 15 and 16 show the life factors at the tight end of the tolerance band for 30-, 50-, 75-, and 100-mm-bore deep-groove and angular-contact ball bearings at the three maximum Hertz stress levels. These plots can be used to estimate the interference-fit life factor at Hertz stress levels between the values analyzed in this article. The curves also show that interference fit has relatively less effect at higher loads, where the unfactored bearing life is shorter. Note that if the bearing or shaft has a different tolerance than the ABEC-5 tolerance considered in this article, the life factor values should be adjusted appropriately.

The life factors found here for deep-groove ball bearings are similar to, but slightly greater than, those reported for cylindrical roller bearings in Oswald et al. (Ref. 10). Note, however, that cylindrical roller bearings have a much higher radial load capacity than ball bearings.

Application of the life factors presented here may result in conservative predictions of bearing life because bearing life calculations and published life values are based on tests that may have included some level of fit. However, the fits used for the tests underlying the life calculations are unreported and thus not known. We have not attempted to account for these unknown fits.

The authors do not recommend that tight interference fits be avoided where operating conditions (speed and vibration levels) demand such fits unless an alternate method for preventing fretting between the bearing race and shaft is provided. Such alternate methods may include splines or keyways.

\section{Effect of Interference Fit on Stress-Life Exponent}

From Lundberg and Palmgren (Ref. 6), the theoretical relationship between the maximum Hertz stress and life in a roller bearing (with line contact) is an inverse eighth power. For ball bearings (with point contact) the relationship is an inverse ninth power where $n=9$ is the stress-life exponent for ball bearings:

$$
L \sim \frac{1}{S_{\max }^{n}}
$$

Interference fits can affect this maximum Hertz stress-life relation. Six curves for maximum Hertz stress versus life are shown in Figure 17 (for five interference fits plus zero fit) on 210 -size ball bearings. For each interference fit, the Hertz stresslife exponent $n$ was calculated. The values for $n$ are also shown in Figure 17.

With no interference fit, the Hertz stress-life exponent $n$ is 9, based on Lundberg and Palmgren (Ref. 6). However, with an average $\mathrm{m} 6$ interference fit, the calculation resulted in $n=8.63$ for deep-groove ball bearings and 8.74 for angularcontact ball bearings. When the results were recalculated based on the tight end of the tolerance band for the $\mathrm{m} 6$ interference fit (not shown in Fig. 17), $n$ was found to be 8.36 and 8.52 for deepgroove and angular-contact ball bearings, respectively. Similar results were obtained for other bearing sizes.

This result of a reduction in the Hertz stress-life exponent is similar to that reported in Oswald et al. (Ref. 10) for cylindrical roller bearings except that for roller bearings, the nominal Hertz stress-life exponent, $n$ is 8 rather than 9 .

This effect on the stress-life exponent can impact the results of accelerated testing on bearings that have a heavy interference fit. If such tests are performed with a heavy load (thus at high Hertz stress) and the test results are then extrapolated to lower stress levels using the usual Hertz stress-life exponent $n=9$, the predicted value of life may be too high, giving a nonconservative design. 

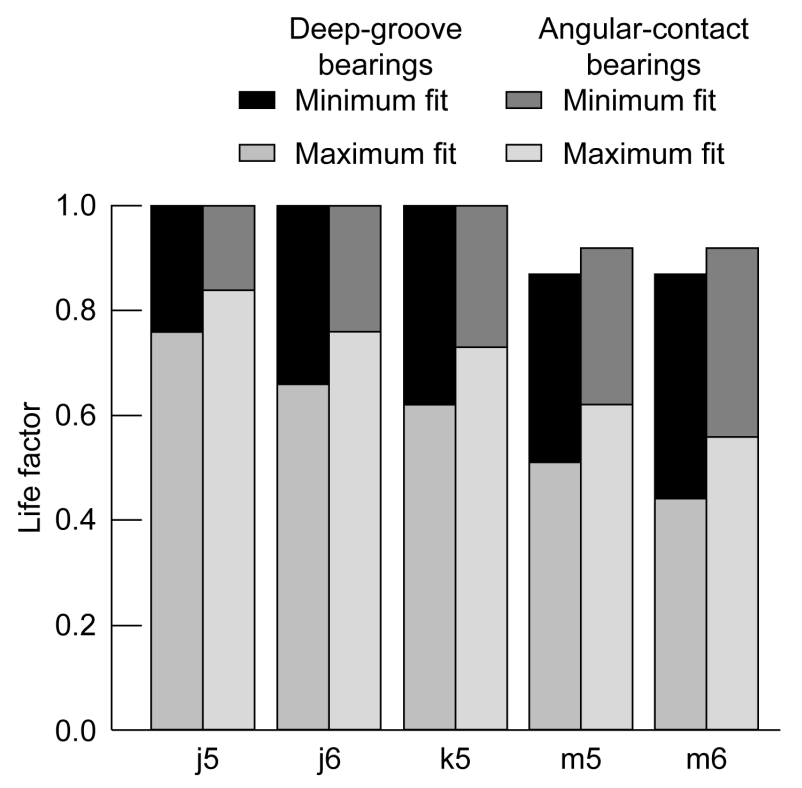

(a)

Fit class

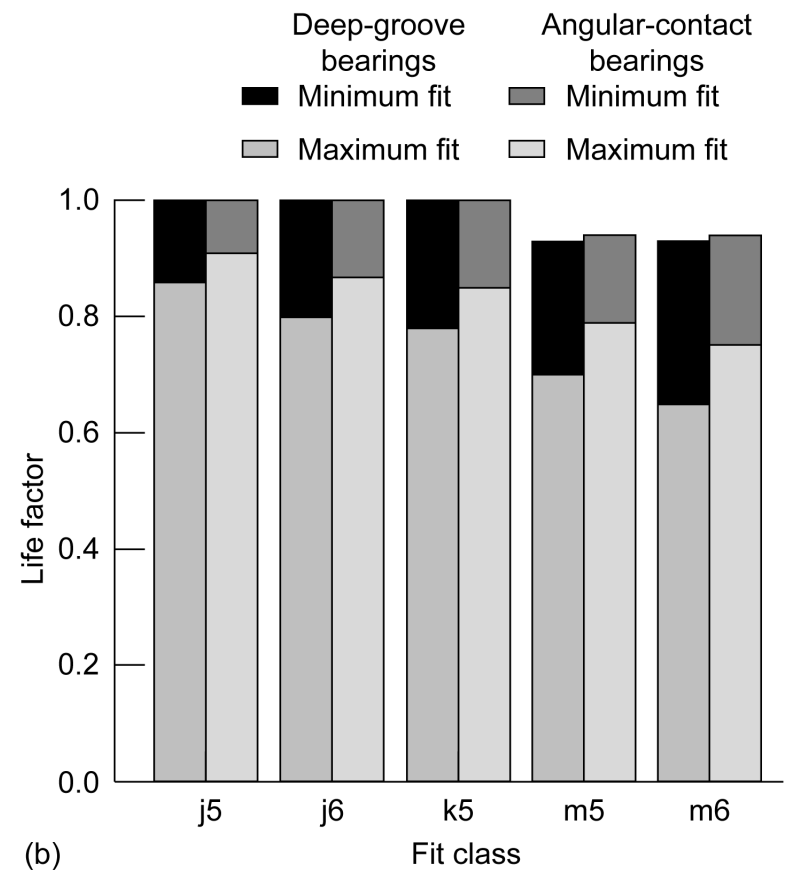

Figure 14.-Life factors for inner-ring interference-fit on 50-mm deep-groove and angular-contact ball bearings at two maximum Hertz stress values. Life factors shown at maximum and minimum fit for $\mathrm{ABEC}-5$ tolerance levels for data averaged from size 1910, 1010, 210, and 310 bearings (Table 3 data without the average fits).(a) $1200 \mathrm{MPa}$ (175 ksi). (b) $2240 \mathrm{MPa}$ (325 ksi). 

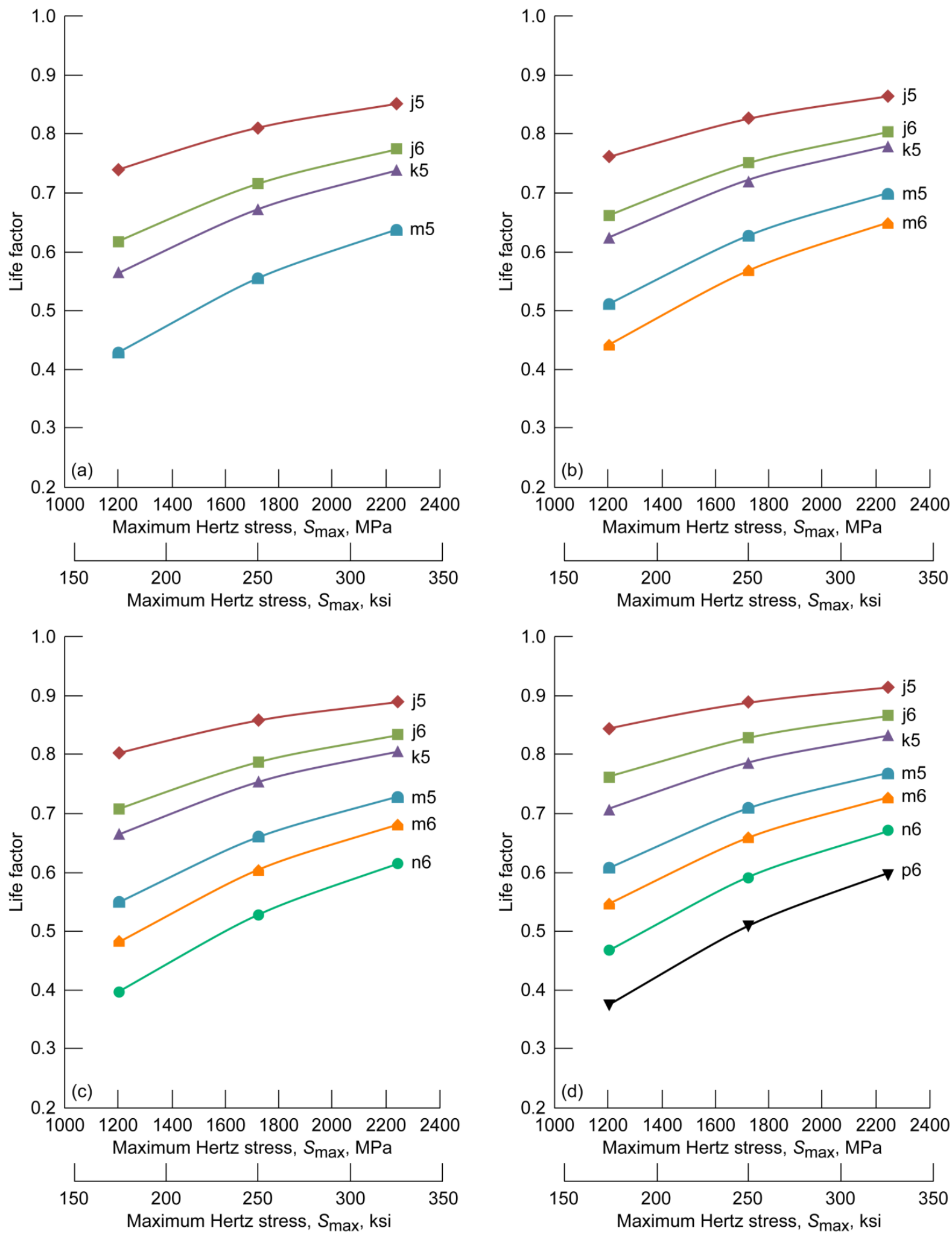

Figure 15.-Life factor versus inner-ring maximum Hertz stress in deep-groove ball bearings for several fit classes. Life factor computed for tight end of ABEC-5 tolerance band for each fit. (a) 30-mm bearings. (b) 50-mm bearings. (c) $75-\mathrm{mm}$ bearings. (d) $100-\mathrm{mm}$ bearings. 

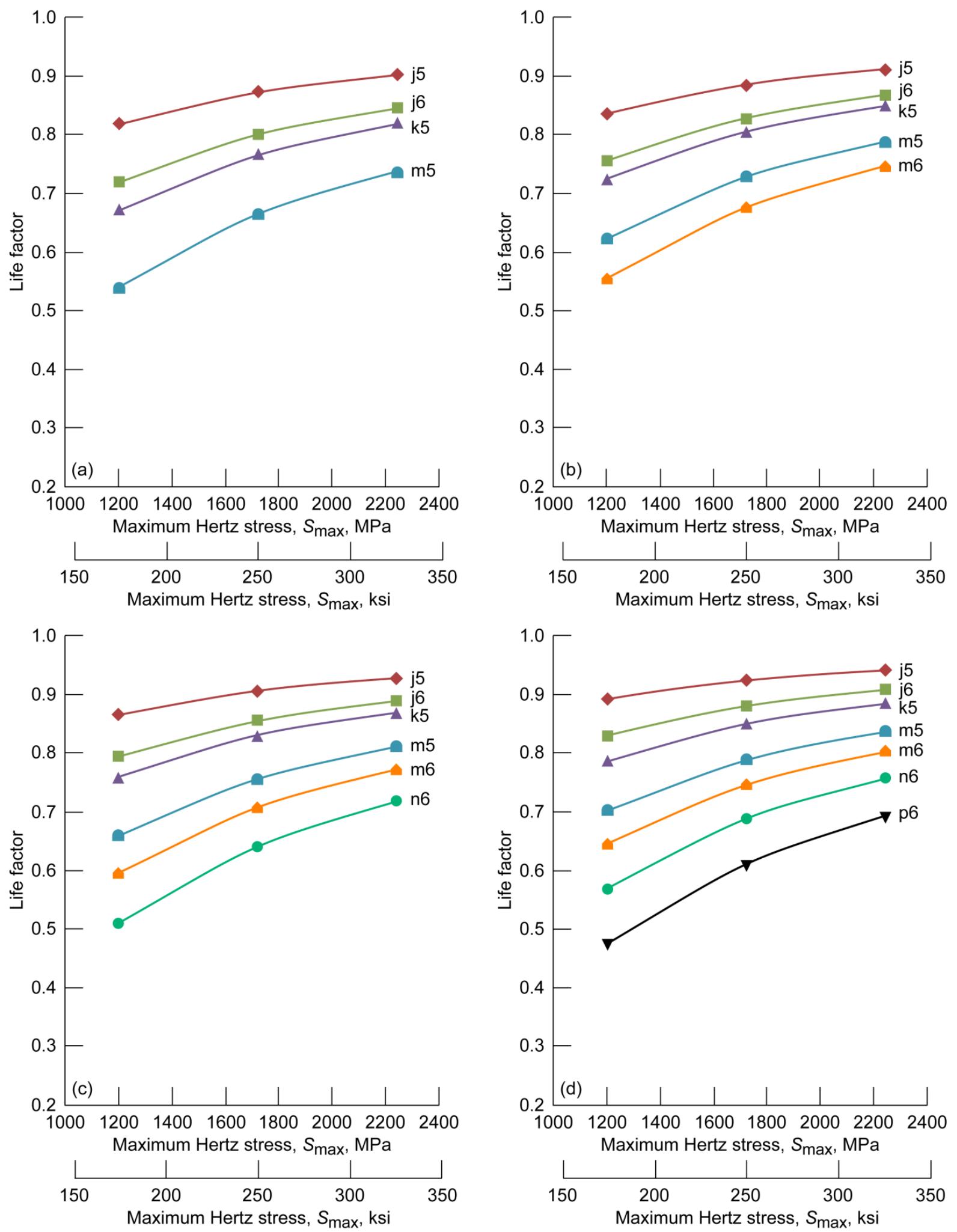

Figure 16.-Life factor versus inner-ring maximum Hertz stress in angular-contact ball bearings for several fit classes. Life factor computed for tight end of ABEC-5 tolerance band for each fit. (a) 30-mm bearings. (b) 50-mm bearings.

(c) $75-\mathrm{mm}$ bearings. (d) $100-\mathrm{mm}$ bearings. 

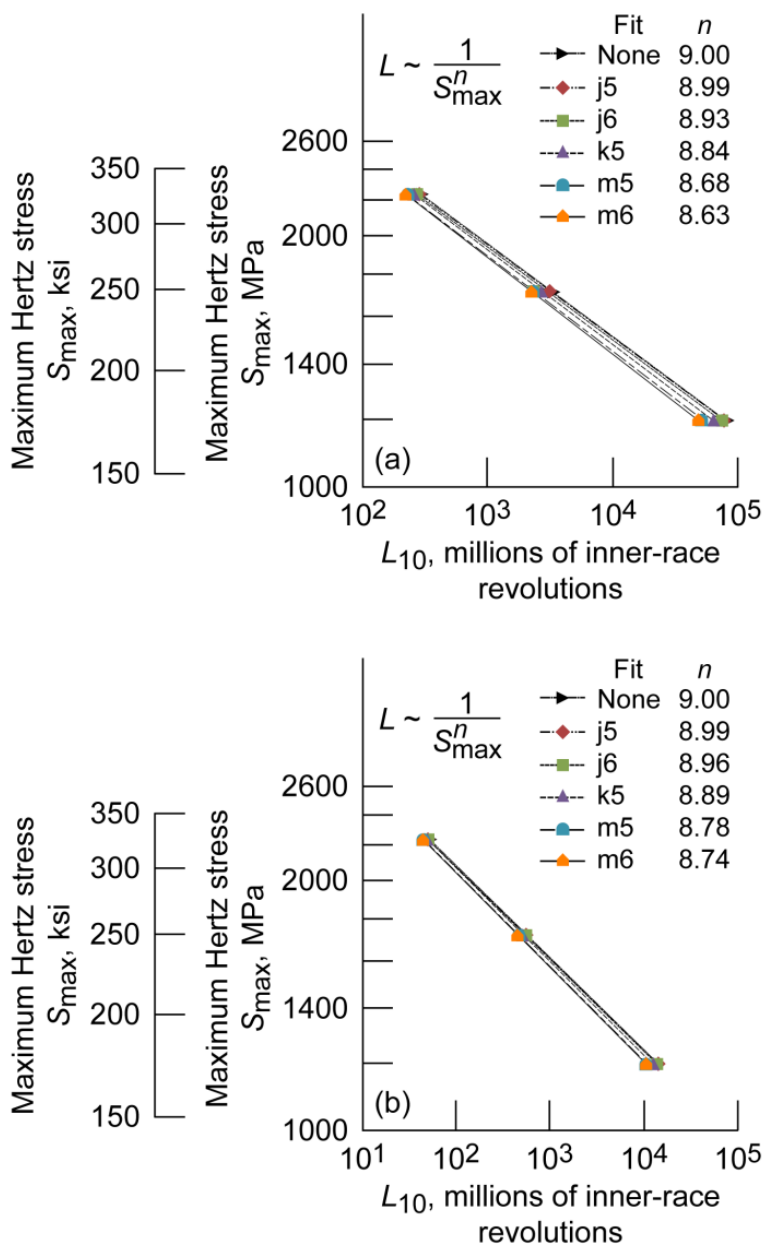

Figure 17.-Relationship between life, $L_{10}$, and maximum Hertz stress, $S_{\max }$, showing effect of interference fit on the stress-life exponent, $n$. (a) 210-size deep-groove ball bearing. (b) 210-size angular-contact ball bearing.

\section{Summary of Results}

The effect of hoop stresses in reducing deep-groove and angular-contact ball bearing fatigue life was determined for various classes of inner-ring interference fit. Calculations were performed for up to seven interference-fit classes for each bearing series. Each fit was taken at the maximum, average, and minimum values within the fit class for ABEC-5 tolerances, thus requiring over 1150 separate analyses.

The hoop stresses were superimposed on the Hertzian principal stresses created by light, normal, and heavy applied radial loads to determine ball bearing fatigue life. The results are presented as life factors for bearings loaded to maximum Hertz stress levels of 1200, 1720, and $2240 \mathrm{MPa}(175,250$, and $325 \mathrm{ksi}$ ) in up to seven fit classes (very light to very heavy) and for bearing accuracy class ABEC-5. If the bearing or shaft has a different tolerance than considered herein, the life factor values should be adjusted appropriately.
The experimental data of Czyzewski (Ref. 3), showing the effect of interference fit on rolling-element fatigue life, were reanalyzed to determine the shear stress-life exponent $c$. This was compared with the analysis reported in this paper.

All calculations are for zero initial internal clearance conditions and assume low speed (below 1 million $D N$ ). Any reduction in internal bearing clearance due to the interference fit would be compensated for by increasing the initial (unmounted) clearance.

Bearing life data as calculated from bearing standards or as found in manufacturers' catalogs are based on tests that presumably would have included interference fits on the rings. However, these fits are unreported and unknown. Therefore, the life factor calculations reported herein are made using the conservative assumption that the bearing life calculations did not include the effect of interference fit.

The life factor for interference fit in low-speed ball bearings can be determined through charts or tables from the maximum Hertz stress, which is easily calculated from the applied radial load and the static-load capacity. The following results were obtained.

(1) Interference fits on the inner bearing ring of a ball bearing can significantly reduce fatigue life. A heavy fit (maximum end of $\mathrm{m} 6$ tolerance band) on a 50 -mm-bore deepgroove ball bearing reduced the fatigue life by 56,43 , and 35 percent from the standard life at maximum Hertz stresses of 1200,1720 , and $2240 \mathrm{MPa}(175,250$, and $325 \mathrm{ksi})$, respectively.

(2) The experimental data of Czyzewski exhibit a shear stress-life exponent $c$ that equals 8.77, in comparison to an assumed value of 9 .

(3) Life factors on thrust-loaded angular-contact ball bearings were higher than on radially loaded deep-groove ball bearings. However, the life of angular-contact bearings for a given maximum Hertz stress is lower than that of deep-groove bearings.

(4) Tighter interference fits produced smaller life factors (i.e., shorter lives). Life factors due to hoop stresses found in this study ranged from 1.00 (no effect) - where there was no interface pressure - to as low as 0.38 (62-percent life reduction) for the maximum $\mathrm{p} 6$ fit on a 100 -mm-bore deep-groove ball bearing and a life factor of 0.47 (53-percent reduction) on a $100-\mathrm{mm}$ angular contact ball bearing at $1200 \mathrm{MPa}(175 \mathrm{ksi})$ maximum Hertz stress.

(5) In general, the life factor was smallest (greatest life reduction) for bearings running under light load where the unfactored life was highest. For any particular bearing size and interference fit, as the maximum Hertz stress on the innerrace was increased, the effect of the hoop stresses on life was reduced, thus increasing the resulting life factor.

(6) Interference fits affected the maximum Hertz stress-life relation. With no interference fit, a Hertz stress-life exponent of $n=9$ was assumed. An m6 middle-of-the-tolerance-band interference fit resulted in $n=8.63$ for deep-groove ball bearings and $n=8.74$ for angular-contact ball bearings. 


\section{Appendix.-Nomenclature}

$A_{\text {sector }} \quad$ Area of bearing ring cross-section removed in grind- $S$ ing the ball track

a Semiwidth of major axis of Hertzian contact area, $\mathrm{mm}$ (in.)

$b$ Semiwidth of minor axis of Hertzian contact area, $\mathrm{mm}$ (in.)

$C_{D} \quad$ Dynamic load capacity, $\mathrm{N}(\mathrm{lbf})$

$C_{\text {or }} \quad$ Static radial load capacity, N (lbf)

$c \quad$ Shear stress-life exponent

$D \quad$ Diameter at the location of the maximum shear stress beneath the surface of the inner race, $\mathrm{mm}$ (in.)

$D N \quad$ Bearing speed parameter: bearing speed in rpm multiplied by the bearing bore diameter in millimeters

$d \quad$ Ball diameter, $\mathrm{mm}$ (in.)

E Young's modulus of elasticity, MPa (ksi)

$e \quad$ Weibull slope or modulus (taken to be 1.11 herein)

$F \quad$ Probability of failure $(1-S)$, fraction or percent

$f_{c} \quad$ Bearing geometry and material coefficient used in Lundberg-Palmgren equation (Lundberg and Palmgren (Ref. 6)). (Standards currently designate this as $f_{c m}$ )

$f_{i} \quad$ Inner race conformity $R_{i} / d$, where $R_{i}$ is the raceway groove radius and $d$ is the ball diameter

$k_{1} \quad$ Conversion constant for Equation (4)

$k_{2} \quad$ Conversion constant for Equation (20)

$k_{3} \quad$ Parameter used to calculate location of maximum shear stress (in the example analyses provided in this paper)

$L \quad$ Life, millions of inner-race revolutions or hours

$L_{10} \quad$ 10-percent life, or life at which 90 percent of a population survives, millions of inner-race revolutions or hours

$(L F)_{h} \quad$ Life factor for hoop stress

$L R \quad$ Life ratio for hoop stress on the inner ring defined in Equation (15)

$n \quad$ Hertz stress-life exponent

$P \quad$ Radial load on bearing, N (lbf)

$p \quad$ Load-life exponent

$p_{i} \quad$ Contact pressure between shaft and inner ring due to interference fit, $\mathrm{MPa}$ (psi)

$R \quad$ Radius of curvature, $\mathrm{mm}$ (in.)

$R^{\prime} \quad$ Ratio of $D_{I R}$ to $d$
$S \quad$ Reliability or probability of survival $(1-F)$, fraction or percent

$S_{\max } \quad$ Maximum Hertz stress, MPa (ksi)

$S_{n} \quad$ Normal stress, MPa (ksi)

$S_{t} \quad$ Tangential stress, MPa (ksi)

$S_{t}^{\prime} \quad$ Tangential stress including hoop stress superimposed on Hertz stress, MPa (ksi)

$u \quad$ Dimensionless depth $(z / b)$ below surface to location of maximum shear stress

$W \quad$ Width of bearing ring, $\mathrm{mm}$ (in)

$X \quad$ Ratio of the lives of the inner and outer races

$z \quad$ Distance below surface to maximum shear stress due to Hertzian load, mm (in.)

a Contact angle, deg

$\Delta \quad$ Diametral interference, $\mathrm{mm}$ (in.)

$\theta \quad$ Sector angle, rad

$v \quad$ Poisson's ratio

$\sigma \quad$ Stress, MPa (ksi)

$\tau \quad$ Shear stress, MPa (ksi)

$\left(\tau_{\max }\right)_{h} \quad$ Maximum shear stress modified by hoop stress, MPa (ksi)

\section{Subscripts}

adj Adjusted life

$B \quad$ Ball set

eff Effective, used to adjust the diameter of the inner ring in Equations (8a) and (8b)

eq Equivalent

$h \quad$ Hoop stress (in tangential or $x$-direction)

$I R \quad$ Inner race of bearing

max Maximum

$n \quad$ Normal direction

$O R \quad$ Outer race of bearing

$r \quad$ residual stress

$S \quad$ Shaft and inner-ring bore

$t \quad$ Tangential direction

$x \quad$ Tangential direction (i.e., in rolling direction)

$y \quad$ Transverse direction

$z \quad$ Normal direction 


\section{References}

1. American Bearing Manufacturers Association (ABMA). (2001), "Shaft and Housing Fits for Metric Radial Ball and Roller Bearings (Except Tapered Roller Bearings) Conforming to Basic Boundary Plan," ANSI/ABMA-7:1995 (R2001), The American Bearing Manufacturers Association, Washington, DC.

2. American Bearing Manufacturers Association (ABMA). (2008), "Radial Bearings of Ball, Cylindrical Roller and Spherical Roller Types-Metric Design," ANSI/ABMA 20-1996 (R2008), The American Bearing Manufacturers Association, Washington, DC.

3. Czyzewski, T. (1975), "Influence of a Tension Stress Field Introduced in the Elastohydrodynamic Contact Zone on Rolling Element Fatigue," Wear, 34, 2, pp. 201-214.

4. Coe, H.H. and Zaretsky, E.V. (1986), "Effect of Interference Fits on Roller Bearing Fatigue Life," ASLE Transactions, 30, 2, pp. 131-140.

5. Jones, A.B. (1946), Analysis of Stress and Deflections, Vol. 1, New Departure Div., General Motors Corp., Bristol, CT.

6. Lundberg, G. and Palmgren, A. (1947), "Dynamic Capacity of Rolling Bearings," Acta Polytech, Mechanical Engineering Series, 1, 3, Stockholm, Sweden.

7. Zaretsky, E.V., ed. (1999), STLE Life Factors for Rolling Bearings, STLE SP-34, 2nd edition, Society of Tribologists and Lubrication Engineers, Park Ridge, IL. ISBN 10: 9993313599 , ISBN 13: 9789993313595.

8. Zaretsky, E.V., Poplawski, J.V. and Root, L.E. (2007), "Reexamination of Ball-Race Conformity Effects on Ball Bearing Life," Tribology Transactions, 50, 3, pp. 336-349.

9. Zaretsky E.V., Coe H.H. and August R. (1997), "Effect of Hoop Stress on Ball Bearing Life Prediction," Tribology Transactions, 40, 1, pp. 91-101.

10. Oswald, F.B., Zaretsky, E.V. and Poplawski, J.V. (2009), "Interference-Fit Life Factors for Roller Bearings," Tribology Transactions, 52, 4, 415-426.

11. Brewe, D. E. and Hamrock, B. J. (1977), "Simplified Solution for Elliptical-Contact Deformation Between Two Elastic Solids," ASME Journal of Lubrication Technology, 99, series F, pp. 485-487.

12. Hamrock, B.J. and Brewe, D. (1983), "Simplified Solution for Stresses and Deformations," ASME Journal of Lubrication Technology, 105, pp. 171-177.

13. Antoine J-F., Visa, C., Sauvey, C. and Abba, G. (2006), “Approximate Analytical Model for Hertzian Elliptical Contact Problems," ASME Journal of Tribology, 128, 3, pp. 660-664. Juvinall, R.C. (1967), Engineering Considerations of Stress Strength and Strain, McGraw Hill, New York, NY. LCCN: 67011208.
15. Weibull, W. (1939), "A Statistical Theory of the Strength of Materials," Ingeniorsvetenskapsakad, Handl., No. 151, Roy. Swed. Inst. Eng. Res, Stockholm, Sweden.

16. Weibull, W. (1939), "Phenomenon of Rupture of Solids," Ingeniorsvetenskapsakad Handl., No. 153, Roy. Swed. Inst. Eng. Res, Stockholm, Sweden.

17. Weibull, W. (1951), "A Statistical Distribution Function of Wide Applicability," Journal of Applied Mechanics, ASME Transactions, 18, 3, pp. 293-297.

18. Poplawski, J.V., Peters, S.M. and Zaretsky, E.V. (2001), "Effect of Roller Profile on Cylindrical Roller Bearing Life PredictionPart I Comparison of Bearing Life Theories," Tribology Transactions, 44, 3, pp. 339-350.

19. Palmgren, A. (1945), Ball and Roller Bearing Engineering, $1^{\text {st }}$ Edition, translation by G. Palmgren and B. Ruley, SKF Industries, Philadelphia, PA.

20. The Timken Company (2004), "Timken Aerospace Design Guide for Precision Metric Ball and Cylindrical Roller Bearings," 3M 0404-06 Order No. 5912, The Timken Company, http://www.timken.com/enus/products/bearings/productlist/aerospace/Documents/aerospace $\%$ 20product \%20design\%20guide.pdf, accessed April 23, 2010.

21. Anti-Friction Bearing Manufacturers Association (AFBMA), (1978), "American National Standard, AFBMA Standard-Load Ratings and Fatigue Life for Ball Bearings," ANSI/AFBMA-Std 9:1978, Anti-Friction Bearing Manufacturers Association, Arlington, VA.

22. American Bearing Manufacturers Association (ABMA). (2000), "Load Ratings and Fatigue Life for Ball Bearings," ANSI/ABMA-9:1990 (R2000), American Bearing Manufacturers Association, Washington, DC.

23. International Organization for Standardization (ISO), (2006), "Rolling Bearings - Static Load Ratings," ISO 76:2006, International Organization for Standardization, Geneva, Switzerland.

24. Poplawski, J. V., Rumbarger, J. H., Peters, S. M., Flower, R. and Galaitis, H. (2002) "Advanced Analysis Package for High Speed Multibearing Shaft Systems: COBRA-AHS," Final report, NASA Contract NAS3-00018.

25. Czyzewski, T. (1975), "Changes in the Stress Field in the Elastohydrodynamic Contact Zone Due to Some Operating Factors and Their Possible Role in the Rolling Contact Fatigue of Cylindrical Surfaces," Wear, 31, 1, pp. 119-140

26. Harris, T.A. (1991), Rolling Bearing Analysis, $3^{\text {rd }}$ Edition, John Wiley \& Sons, Inc., New York, NY, ISBN 0-471-51349-0. 


\begin{tabular}{|c|c|c|c|c|c|}
\hline \multicolumn{5}{|c|}{ REPORT DOCUMENTATION PAGE } & $\begin{array}{c}\text { Form Approved } \\
\text { OMB No. 0704-0188 }\end{array}$ \\
\hline \multicolumn{6}{|c|}{ 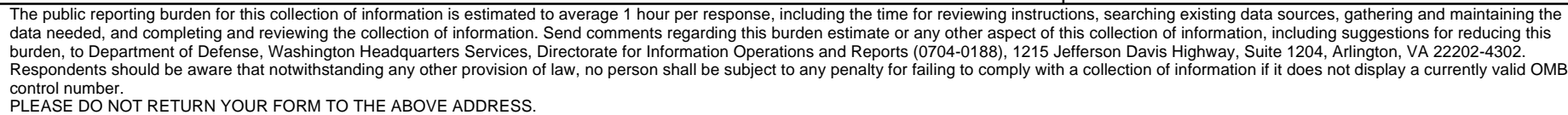 } \\
\hline \multicolumn{2}{|c|}{$\begin{array}{l}\text { 1. REPORT DATE (DD-MM-YYYY) } \\
01-11-2010\end{array}$} & \multicolumn{3}{|c|}{$\begin{array}{l}\text { 2. REPORT TYPE } \\
\text { Technical Memorandum }\end{array}$} & 3. DATES COVERED (From - To) \\
\hline \multirow{3}{*}{\multicolumn{5}{|c|}{$\begin{array}{l}\text { 4. TITLE AND SUBTITLE } \\
\text { Interference-Fit Life Factors for Ball Bearings }\end{array}$}} & 5a. CONTRACT NUMBER \\
\hline & & & & & 5b. GRANT NUMBER \\
\hline & & & & & 5c. PROGRAM ELEMENT NUMBER \\
\hline \multirow{3}{*}{\multicolumn{5}{|c|}{$\begin{array}{l}\text { 6. AUTHOR(S) } \\
\text { Oswald, Fred, B.; Zaretsky, Erwin, V.; Poplawski, Joseph, V. }\end{array}$}} & 5d. PROJECT NUMBER \\
\hline & & & & & 5e. TASK NUMBER \\
\hline & & & & & $\begin{array}{l}\text { 5f. WORK UNIT NUMBER } \\
\text { WBS 877868.02.07.03.04.01 }\end{array}$ \\
\hline \multicolumn{5}{|c|}{$\begin{array}{l}\text { 7. PERFORMING ORGANIZATION NAME(S) AND ADDRESS(ES) } \\
\text { National Aeronautics and Space Administration } \\
\text { John H. Glenn Research Center at Lewis Field } \\
\text { Cleveland, Ohio 44135-3191 }\end{array}$} & $\begin{array}{l}\text { 8. PERFORMING ORGANIZATION } \\
\text { REPORT NUMBER } \\
\text { E-17001-1 }\end{array}$ \\
\hline \multirow{2}{*}{\multicolumn{5}{|c|}{$\begin{array}{l}\text { 9. SPONSORING/MONITORING AGENCY NAME(S) AND ADDRESS(ES) } \\
\text { National Aeronautics and Space Administration } \\
\text { Washington, DC 20546-0001 }\end{array}$}} & $\begin{array}{l}\text { 10. SPONSORING/MONITOR'S } \\
\text { ACRONYM(S) } \\
\text { NASA }\end{array}$ \\
\hline & & & & & $\begin{array}{l}\text { 11. SPONSORING/MONITORING } \\
\text { REPORT NUMBER } \\
\text { NASA/TM-2010-216913 }\end{array}$ \\
\hline \multicolumn{6}{|c|}{$\begin{array}{l}\text { 12. DISTRIBUTION/AVAILABILITY STATEMENT } \\
\text { Unclassified-Unlimited } \\
\text { Subject Category: } 37 \\
\text { Available electronically at http://gltrs.grc.nasa.gov } \\
\text { This publication is available from the NASA Center for AeroSpace Information, 443-757-5802 }\end{array}$} \\
\hline \multicolumn{6}{|c|}{$\begin{array}{l}\text { 13. SUPPLEMENTARY NOTES } \\
\text { Submitted to Tribology Transactions. }\end{array}$} \\
\hline \multicolumn{6}{|c|}{$\begin{array}{l}\text { The effect of hoop stresses on the rolling-element fatigue life of angular-contact and deep-groove ball bearings was determined for common } \\
\text { inner-ring interference fits at the ABEC- } 5 \text { tolerance level. The analysis was applied to over } 1150 \text { bearing configurations and load cases. } \\
\text { Hoop stresses were superimposed on the Hertzian principal stresses created by the applied bearing load to calculate the inner-race maximum } \\
\text { shearing stress. The resulting fatigue life of the bearing was recalculated through a series of equations. The reduction in the fatigue life is } \\
\text { presented as life factors that are applied to the unfactored bearing life. The life factors found in this study ranged from } 1.00 \text { (no life } \\
\text { reduction)--where there was no net interface pressure--to a worst case of } 0.38 \text { (a } 62 \text {-percent life reduction). For a given interference fit, the } \\
\text { reduction in life is different for angular-contact and deep-groove ball bearings. Interference fits also affect the maximum Hertz stress-life } \\
\text { relation. Experimental data of Czyzewski, showing the effect of interference fit on rolling-element fatigue life, were reanalyzed to determine } \\
\text { the shear stress-life exponent. The Czyzewski data shear stress-life exponent } c \text { equals } 8.77 \text {, compared with the assumed value of } 9 \text {. Results } \\
\text { are presented as tables and charts of life factors for angular-contact and deep-groove ball bearings with light, normal, and heavy loads and } \\
\text { interference fits ranging from extremely light to extremely heavy. }\end{array}$} \\
\hline \multicolumn{6}{|c|}{$\begin{array}{l}\text { 15. SUBJECT TERMS } \\
\text { Interface fit; Ball bearings; Rolling bearings; Fatigue analysis; Contacts; Life prediction methods; Maintenance; Rolling-contact } \\
\text { fatigue; Wear; Stress; Analysis }\end{array}$} \\
\hline \multicolumn{3}{|c|}{ 16. SECURITY CLASSIFICATION OF: } & $\begin{array}{l}\text { 17. LIMITATION OF } \\
\text { ABSTRACT }\end{array}$ & $\begin{array}{l}\text { 18. NUMBER } \\
\text { OF }\end{array}$ & $\begin{array}{l}\text { 19a. NAME OF RESPONSIBLE PERSON } \\
\text { STI Help Desk (email:help@sti.nasa.gov) }\end{array}$ \\
\hline $\begin{array}{l}\text { a. REPORT } \\
\text { U }\end{array}$ & $\begin{array}{l}\text { b. ABSTRACT } \\
\text { U }\end{array}$ & $\begin{array}{l}\text { c. THIS } \\
\text { PAGE } \\
\text { U }\end{array}$ & UU & $\begin{array}{l}\text { PAGES } \\
30\end{array}$ & $\begin{array}{l}\text { 19b. TELEPHONE NUMBER (include area code) } \\
443-757-5802\end{array}$ \\
\hline
\end{tabular}



